This is a preprint or reprint of a paper intended for presentation at a conference. Because changes may be made before formal

publication, this is made available with the understanding that it will not be cited or reproduced without the permission of the author.

\title{
AIAA 99-2777
}

Pulsating Hydrodynamic Instability in a Dynamic Model of Liquid-Propellant Combustion

Stephen B. Margolis

Combustion Research Facility

Sandia National Laboratories

Livermore, California 94551-0969 USA

\section{5th AIAA/ASME/SAE/ASEE Joint Propulsion Conference and Exhibit 20-24 June 1999 Los Angeles, California}

For permission to copy or to republish, contact the American Institute of Aeronautics and Astronautics, 1801 Alexander Bell Drive, Suite 500, Reston, VA, 20191-4344. 
AIAA-99-2777

\title{
PULSATING HYDRODYNAMIC INSTABILITY IN A DYNAMIC MODEL OF LIQUID-PROPELLANT COMBUSTION*
}

\author{
STEPHEN B. MARGOLIS \\ Combustion Research Facility \\ Sandia National Laboratories \\ Livermore, California 94551-0969
}

\begin{abstract}
Hydrodynamic (Landau) instability in combustion is typically associated with the onset of wrinkling of a flame surface, corresponding to the formation of steady cellular structures as the stability threshold is crossed. In the context of liquidpropellant combustion, such instability has recently been shown to occur for critical values of the pressure sensitivity of the burning rate and the disturbance wavenumber, significantly generalizing previous classical results for this problem that assumed a constant normal burning rate. Additionally, however, a pulsating form of hydrodynamic instability has been shown to occur as well, corresponding to the onset of temporal oscillations in the location of the liquid/gas interface. In the present work, we consider the realistic influence of a nonzero temperature sensitivity in the local burning rate on both types of stability thresholds. It is found that for sufficiently small values of this parameter, there exists a stable range of pressure sensitivities for steady, planar burning such that the classical cellular form of hydrodynamic instability and the more recent pulsating form of hydrodynamic instability can each occur as the corresponding stability threshold is crossed. For larger thermal sensitivities, however, the pulsating stability boundary evolves into a C-shaped curve in the disturbance-wavenumber/ pressure-sensitivity plane, indicating loss of stability to pulsating perturbations for all sufficiently large disturbance wavelengths. It is thus concluded, based on characteristic parameter values, that an equally likely form of hydrodynamic instability in liquid-propellant combustion is of a nonsteady, long-wave nature, distinct from the steady, cellular form originally predicted by Landau.
\end{abstract}

\footnotetext{
*This paper is declared a work of the U.S. Government and is not subject to copyright protection in the United States.
}

\section{Introduction}

The notion of hydrodynamic instability in combustion originated with Landau's seminal study of premixed flame propagation. ${ }^{1}$ In that work, it was postulated that a flame could be represented by a surface of discontinuity, with an appropriate density jump across the flame surface, and the normal equations of hydrodynamics governing the flow on either side of the flame surface. Assuming that this surface propagated normal to itself with constant speed, it was then determined through a straightforward linear stability analysis that a premixed gaseous flame was intrinsically unstable to steady (cellular) disturbances whenever, as is generally the case, the density on the burned side of the front was less than that of the unburned mixture. Since this result is at variance with experimental observations of steady, planar laboratory flames, it has sparked numerous other theoretical investigations that have sought to resolve this paradox, through both the inclusion of additional effects and the analysis of less phenomenological combustion models. Today, this specific form of hydrodynamic instability, generally referred to as Landau instability, plays a central role in the study of flame/flow interactions. Though Landau's analysis essentially collapsed all thermal effects to the change in density across the front, Landau instability is nonetheless implicitly associated with the thermal expansion of the gas arising from the heat release during combustion. Indeed, this instability persists even when the entire flame structure is resolved, ${ }^{2,3}$ although it is now recognized as a predominantly long-wave cellular instability that is subject to various stabilizing influences for disturbances corresponding to larger wavenumbers.

A related problem briefly addressed at the end of Landau's original study (for which a more standard and less controversial type of result was obtained), and the one of interest here, was a modest modification of the flame-propagation problem just described. In this modified problem, the unburned 
mixture was taken to be a liquid propellant while the burned region again consisted of gaseous products. The physical existence of a liquid/gas interface led to the inclusion of additional physics in the model, namely surface tension at the interface and the influence of gravitational acceleration (downward propagation was assumed). As a result, and in contrast to strictly gaseous flame propagation, a stability criterion was derived such that the liquid/gas interface was either hydrodynamically stable or unstable in the Landau (cellular) sense depending on whether the product of the gravitational acceleration and the coefficient of surface tension was greater or less than a critical value. This result was later extended by Levich, ${ }^{4}$ who considered the effects of (liquid) viscosity in lieu of surface tension and obtained a similar result for the product of the gravitational acceleration and the viscosity of the liquid. These classical models of liquid-propellant combustion, despite their relative simplicity, have long served as seminal examples that correctly describe the onset of (cellular) hydrodynamic instability in reactive systems.

Although the assumption of a thin reaction region remains a frequently valid and useful simplification in many combustion studies, the classical assumption of a constant normal burning rate has long been regarded as a probable oversimplification when applied to the problem of combustion instability. Early attempts at modification began with the phenomenological assumption of a linear relationship between the burning rate and the flame curvature, ${ }^{5}$ while more modern approaches have employed sophisticated asymptotic methods to analyze the flame structure and to derive formal expressions for the burning rate as a function of local conditions at the combustion front, for both gaseous and condensed combustion waves. ${ }^{2,3,6,7}$ In propellant combustion, on the other hand, it has long been customary to experimentally measure the pressure response, or pressure sensitivity, of the burning rate, as well as (to a lesser extent), its temperature sensitivity. Although asymptotic models of propellant combustion that resolve the combustion-wave structure can be developed under various approximations, ${ }^{8-10}$ it remains true nonetheless that the direct assumption of a combustion surface that propagates according to a prescribed burning-rate law, such as a phenomenological law based on experimental observations, allows one to circumvent the intricacies of the combustion region and to impose fewer restrictive assumptions on the hydrodynamic model in the unburned and burned regions.

Accordingly, for the case of liquid-propellant combustion, the classical Landau/Levich hydrody- namic models may be combined and extended to account for a dynamic dependence, absent in the original formulations, of the instantaneous burning rate on the local pressure and temperature fields. ${ }^{11,12}$ Although exact expressions for the neutral stability boundaries in question can be obtained in specific limiting cases, more general results may be obtained by exploiting the realistic smallness of the gas-to-liquid density ratio $\rho$. Thus, neglecting thermal coupling effects, an asymptotic analytical expression was derived for the cellular stability boundary $A_{p}(k)$, where $A_{p}$ denotes the pressure sensitivity of the burning rate and $k$ is the wavenumber of the disturbance. ${ }^{13}$ The results demonstrate explicitly the stabilizing effect of gravity on long-wave disturbances, the stabilizing effect of viscosity (both liquid and gas) and surface tension on short-wave perturbations, and the instability associated with intermediate wavenumbers for critical negative values of $A_{p}$. In the limiting case of weak gravity, it was shown that hydrodynamic instability in liquidpropellant combustion is a long-wave instability phenomenon, whereas at normal gravity, this instability is first manifested through $O(1)$ wavenumber disturbances. It was also demonstrated that, in general, surface tension and the viscosity of both the liquid and gas phases each produce comparable stabilizing effects in the large-wavenumber regime, thereby providing important modifications to the previous classical analyses in which one or more of these effects was neglected. For $A_{p}=0$, the Landau/Levich results are recovered from the new model in appropriate limiting cases, although, in the realistic limit of small $\rho$, this corresponds to a hydrodynamically unstable parameter regime. ${ }^{1,4,13}$

In addition to the classical cellular form of hydrodynamic stability described thus far, there exists in this extended model a pulsating form of hydrodynamic instability corresponding to the loss of stability of the steady, planar solution to time-dependent perturbations. ${ }^{14}$ This occurs for negative values of the pressure-sensitivity parameter $A_{p}$, and is therefore absent from the original Landau/Levich models. Nonetheless, its existence in the extended model results in a stable band of negative pressure sensitivities bounded above by the classical Landau type of hydrodynamic instability, and below by the pulsating form of hydrodynamic instability just described. A stable range of negative pressure sensitivities is applicable, for example, to certain types of hydroxylammonium nitrate (HAN)-based liquid propellants at low pressures for which nonsteady modes of combustion have been observed..$^{15}$ While it is possible that such nonsteady combustion may correspond to 
secondary and higher-order bifurcations above the cellular boundary, ${ }^{16}$ it may also be a manifestation of the pulsating form of hydrodynamic instability just described.

In the present work, ${ }^{18,19}$ we explore the effects of incorporating a nonzero temperature sensitivity into our previous asymptotic analyses performed in the limit of small gas-to-liquid density ratios. ${ }^{13,14}$ This entails a coupling of the energy equation for temperature to the previous purely hydrodynamic problem, and leads to a significant modification to the pulsating boundary such that, for sufficiently large values of the temperature-sensitivity parameter, liquid-propellant combustion can become intrinsically unstable to this newer form of hydrodynamic instability.

\section{The Mathematical Model and its Basic Solution}

The extended mathematical model referred to above was described previously, ${ }^{13}$ but is briefly summarized here for completeness. In particular, it is assumed, as in the classical models, that there is no distributed reaction in either the liquid or gas phases, but that there exists either a pyrolysis reaction or an exothermic decomposition at the liquid/gas interface that depends on local conditions there. For simplicity, it is assumed that within the liquid and gas phases separately, the various physical properties are constants, with appropriate jumps in these quantities across the phase boundary.

The nondimensional location of the downwardpropagating liquid/gas interface, which is a function of space and time, is denoted by $x_{3}=\Phi_{s}\left(x_{1}, x_{2}, t\right)$. where $x_{3}$ is the vertical coordinate and the adopted coordinate system is fixed with respect to the stationary liquid at $x_{3}=-\infty$. Then, in the moving coordinate system $x=x_{1}, y=x_{2}, z=x_{3}$ $\Phi_{s}\left(x_{1}, x_{2}, t\right)$, in terms of which the liquid/gas interface always lies at $z=0$, the complete formulation of the problem is given by conservation of mass, energy and momentum within each phase as

$$
\begin{gathered}
\nabla \cdot \mathrm{v}=0, \quad z \neq 0, \\
\frac{\partial \Theta}{\partial t}-\frac{\partial \Phi_{s}}{\partial t} \frac{\partial \Theta}{\partial z}+\mathrm{v} \cdot \nabla \Theta=\left\{\begin{array}{c}
1 \\
\lambda
\end{array}\right\} \nabla^{2} \Theta, \quad z>0, \\
\frac{\partial \mathrm{v}}{\partial t}-\frac{\partial \Phi_{s}}{\partial t} \frac{\partial \mathrm{v}}{\partial z}+(\mathrm{v} \cdot \nabla) \mathrm{v}=\left(0,0,-F r^{-1}\right) \\
-\left\{\begin{array}{c}
1 \\
\rho^{-1}
\end{array}\right\} \nabla p+\left\{\begin{array}{c}
P_{l} \\
\lambda P r_{g}
\end{array}\right\} \nabla^{2} \mathrm{v}, \quad z>0,
\end{gathered}
$$

where $\mathrm{v}, \Theta$ and $p$ denote velocity, temperature and pressure, respectively, $P r_{l}$ and $P r_{g}$ denote the liquid and gas-phase Prandtl numbers, $\rho, \lambda$ and $c$ (used below) are the gas-to-liquid density, thermal diffusivity and heat-capacity ratios, and $F r$ is the Froude number. These nondimensional variables have been defined in terms of their dimensional counterparts (denoted by tildes) as

$$
\begin{aligned}
& \Theta=\frac{\tilde{T}-\tilde{T}_{u}}{\tilde{T}_{a}-\tilde{T}_{u}}, \quad \mathbf{v}=\frac{\tilde{\mathbf{v}}}{\tilde{U}}, \quad p=\frac{\bar{p}}{\tilde{\rho}_{l} \tilde{U}^{2}}, \\
& \Phi_{s}=\frac{\tilde{\Phi}_{s} \tilde{U}}{\tilde{\lambda}_{l}}, \quad t=\frac{\tilde{t} \bar{U}^{2}}{\tilde{\lambda}_{l}}, \quad x_{i}=\frac{\tilde{x}_{i} \tilde{U}}{\tilde{\lambda}_{l}},
\end{aligned}
$$

where $\bar{U}$ is the reference propagation speed of the interface for the case of steady, planar deflagration. The nondimensional parameters, some of which first appear below in the conditions at the gas/liquid interface, have been defined as

$$
\begin{aligned}
& \rho=\frac{\tilde{\rho}_{g}}{\bar{\rho}_{l}}, \quad \sigma_{u}=\frac{\bar{T}_{u}}{\tilde{T}_{a}}, \quad \lambda=\frac{\bar{\lambda}_{g}}{\bar{\lambda}_{l}}, \\
& c=\frac{\bar{c}_{g}}{\bar{c}_{l}}, \quad F r=\frac{\tilde{U}^{3}}{\bar{g} \bar{\lambda}_{l}}, \quad \gamma=\frac{\bar{\gamma}}{\bar{\rho}_{l} \bar{\lambda}_{l} \bar{U}}, \\
& \operatorname{Pr}_{l}=\frac{\bar{\nu}_{l}}{\bar{\lambda}_{l}}, \quad P r_{g}=\frac{\bar{\nu}_{g}}{\bar{\lambda}_{g}},
\end{aligned}
$$

where $\bar{T}_{u}$ is the unburned (liquid) temperature at $z=-\infty$ and $\tilde{T}_{a}$ is the adiabatic burned (gas) temperature at $z=+\infty, \bar{\gamma}$ is the coefficient of surface tension for the liquid surface, and $\tilde{\nu}_{l}$ and $\vec{\nu}_{g}$ are the liquid and gas-phase kinematic viscosities. We note that the inverse Froude number $\mathrm{Fr}^{-1}$ represents the nondimensional gravitational acceleration and that $\rho \lambda P r_{g}=\mu P r_{l}$, where $\mu=\bar{\mu}_{g} / \bar{\mu}_{l}$ is the gasto-liquid viscosity ratio. The nondimensional mass burning rate appearing in Eq. (9) below is defined by $A\left(\left.p\right|_{z=0^{+}},\left.\Theta\right|_{z=0}\right)=\tilde{A}\left(\left.\tilde{p}\right|_{\tilde{x}_{3}=\bar{\Phi}^{+}},\left.\tilde{T}\right|_{\bar{x}_{3}=\tilde{\Phi}_{0}}\right) / \tilde{\rho}_{l} \tilde{U}$, and is assumed to be functionally dependent on the local pressure and temperature at the liquid/gas interface. By definition, $A=1$ for the case of steady, planar burning, but perturbations in pressure and/or termperature result in corresponding perturbations in the local mass burning rate.

Equations (1) - (3) are subject to the boundary conditions

$$
\begin{aligned}
& \mathbf{v}=0, \Theta=0 \text { at } z=-\infty \\
& \Theta=1 \text { at } z=+\infty,\left.\quad \Theta\right|_{z=0^{-}}=\left.\Theta\right|_{z=0^{+}}
\end{aligned}
$$

and appropriate jump and continuity conditions at the liquid/gas interface. The latter consist of continuity of the transverse velocity components (noslip),

$$
\hat{\mathbf{n}}_{\mathbf{s}} \times \mathbf{v}_{-}=\hat{\mathbf{n}}_{\mathbf{s}} \times \mathbf{v}_{+},
$$


where $v_{ \pm}=\left.v\right|_{z=0^{ \pm}}$, conservation of (normal) mass flux,

$$
\hat{\mathbf{n}}_{\mathbf{s}} \cdot\left(\mathbf{v}_{-}-\rho \mathbf{v}_{+}\right)=(1-\rho) S\left(\Phi_{s}\right) \frac{\partial \Phi_{s}}{\partial t}
$$

the mass burning rate (pyrolysis) law,

$$
\hat{\mathbf{n}}_{\mathbf{s}} \cdot \mathbf{v}_{-}-S\left(\Phi_{s}\right) \frac{\partial \Phi}{\partial t}=A\left(\left.p\right|_{z=0^{+}},\left.\Theta\right|_{z=0}\right)
$$

conservation of flux of the normal and transverse components of momentum,

$$
\begin{aligned}
&\left.p\right|_{z=0^{-}}-\left.p\right|_{z=0^{+}} \\
&=\hat{\mathbf{n}}_{\mathbf{s}} \cdot\left[\rho \mathbf{v}_{+}\left(\hat{\mathbf{n}}_{\mathbf{s}} \cdot \mathbf{v}_{+}\right)-\mathbf{v}_{-}\left(\hat{\mathbf{n}}_{\mathbf{s}} \cdot \mathbf{v}_{-}\right)\right.\left.\quad-\rho \lambda P r_{g} \mathbf{e}_{+} \cdot \hat{\mathbf{n}}_{\mathbf{s}}+P r_{l} \mathbf{e}_{-} \cdot \hat{\mathbf{n}}_{\mathbf{s}}\right] \\
&+ \hat{\mathbf{n}}_{\mathbf{s}} \cdot\left(\mathbf{v}_{-}-\rho \mathbf{v}_{+}\right) S\left(\Phi_{s}\right) \frac{\partial \Phi_{s}}{\partial t} \\
&-\gamma S^{3}\left(\Phi_{s}\right)\left\{\frac{\partial^{2} \Phi_{s}}{\partial x^{2}}\left[1+\left(\frac{\partial \Phi_{s}}{\partial y}\right)^{2}\right]\right. \\
&\left.+\frac{\partial^{2} \Phi_{s}}{\partial y^{2}}\left[1+\left(\frac{\partial \Phi_{s}}{\partial x}\right)^{2}\right]-2 \frac{\partial \Phi_{s}}{\partial x} \frac{\partial \Phi_{s}}{\partial y} \frac{\partial^{2} \Phi_{s}}{\partial x \partial y}\right\} \\
& \hat{\mathbf{n}}_{\mathbf{s}} \times\left[\rho \mathbf{v}_{+}\left(\hat{\mathbf{n}}_{\mathbf{s}} \cdot \mathbf{v}_{+}\right)-\mathbf{v}_{-}\left(\hat{\mathbf{n}}_{\mathbf{s}} \cdot \mathbf{v}_{-}\right)\right. \\
&\left.\quad+\left(\mathbf{v}_{-}-\rho \mathbf{v}_{+}\right) S\left(\Phi_{s}\right) \frac{\partial \Phi_{s}}{\partial t}\right] \\
&=\hat{\mathbf{n}}_{\mathbf{s}} \times\left(\rho \lambda P r_{g} \mathbf{e}_{+} \cdot \hat{\mathbf{n}}_{\mathbf{s}}-\operatorname{Pr}_{l} \mathbf{e}_{-} \cdot \hat{\mathbf{n}}_{\mathbf{s}}\right),
\end{aligned}
$$

and conservation of heat flux

$$
\begin{aligned}
& \hat{\mathbf{n}}_{\mathbf{s}} \cdot(\left(\left.c \rho \lambda \nabla \Theta\right|_{z=0^{+}}-\left.\nabla \Theta\right|_{z=0^{-}}\right) \\
&=\hat{\mathbf{n}}_{\mathbf{s}} \cdot\left[\left.\left(c \rho \mathbf{v}_{+}-\mathbf{v}_{-}\right) \Theta\right|_{z=0}+\hat{c}\left(\sigma_{u} \rho \mathbf{v}_{+}-\mathbf{v}_{-}\right)\right] \\
& \quad+\left[\left.(1-c \rho) \Theta\right|_{z=0}+\hat{c}\left(1-\sigma_{u} \rho\right)\right] S\left(\Phi_{s}\right) \frac{\partial \Phi_{s}}{\partial t}
\end{aligned}
$$

where $\hat{c}=c /\left(1-\sigma_{u}\right)$, $\mathbf{e}$ is the rate-of-strain tensor $\left(\mathbf{e}_{ \pm}=\left.\mathbf{e}\right|_{z=0^{ \pm}}\right), \gamma$ is the surface-tension coefficient, $\sigma_{u}$ is the unburned-to-burned temperature ratio, and $S\left(\Phi_{s}\right)$ and the unit normal $\hat{\mathbf{n}}_{\mathbf{s}}$ are defined as

$$
\begin{aligned}
& S\left(\Phi_{s}\right)=\left[1+\left(\partial \Phi_{s} / \partial x\right)^{2}+\left(\partial \Phi_{s} / \partial y\right)^{2}\right]^{-1 / 2} \\
& \hat{n}_{\mathbf{s}}=\left(-\partial \Phi_{s} / \partial x,-\partial \Phi_{s} / \partial y, 1\right) S\left(\Phi_{s}\right)
\end{aligned}
$$

Here, the factor multiplying $\gamma$ in Eq. (10) is the curvature $-\nabla \cdot \hat{\mathbf{n}}_{\mathbf{s}}$ of the liquid/gas interface in the moving coordinate system, and the corresponding expressions for both the gradient operator $\nabla$ and the Laplacian $\nabla^{2}$ in this system are given by

$$
\nabla=\left(\frac{\partial}{\partial x}-\frac{\partial \Phi_{s}}{\partial x} \frac{\partial}{\partial z}, \frac{\partial}{\partial y}-\frac{\partial \Phi_{s}}{\partial y} \frac{\partial}{\partial z}, \frac{\partial}{\partial z}\right),
$$

$$
\begin{gathered}
\nabla^{2}=\frac{\partial^{2}}{\partial x^{2}}+\frac{\partial^{2}}{\partial y^{2}}+\left[1+\left(\frac{\partial \Phi_{s}}{\partial x}\right)^{2}+\left(\frac{\partial \Phi_{s}}{\partial y}\right)^{2}\right] \frac{\partial^{2}}{\partial z^{2}} \\
-2 \frac{\partial \Phi_{s}}{\partial x} \frac{\partial^{2}}{\partial x \partial z}-2 \frac{\partial \Phi_{s}}{\partial y} \frac{\partial^{2}}{\partial y \partial z} \\
-\left(\frac{\partial^{2} \Phi_{s}}{\partial x^{2}}+\frac{\partial^{2} \Phi_{s}}{\partial y^{2}}\right) \frac{\partial}{\partial z}
\end{gathered}
$$

However, the vector $\mathbf{v}$ still denotes the velocity with respect to the $\left(x_{1}, x_{2}, x_{3}\right)$ coordinate system.

We observe that the thermal and hydrodynamic fields are coupled through the temperature dependence of the mass burning rate $A$ appearing in Eq. (9). When $A$ is assumed to depend on pressure only, the strictly hydrodynamic problem for $p, \mathrm{v}$ and $\Phi_{s}$ can be analyzed separately $[13,14]$. In the present work, we wish to focus on this more extended coupling to determine how the hydrodynamic stability boundaries are modified when the local burning rate depends on temperature as well. In connection with this we remark that although the mass burning rate $A$ may be typically decomposed as

$$
\begin{aligned}
& A\left(\left.p\right|_{z=0^{+}},\left.\Theta\right|_{z=0}\right) . \\
& =\hat{A}\left(\left.p\right|_{z=0^{+}},\left.\Theta\right|_{z=0}\right) \exp \left[\frac{N\left(1-\sigma_{u}\right)\left(\left.\Theta\right|_{z=0}-1\right)}{\sigma_{u}+\left.\left(1-\sigma_{u}\right) \Theta\right|_{z=0}}\right],
\end{aligned}
$$

where $\hat{A}$ is a rate coefficient and $N=\tilde{E} / \bar{R}^{\circ} \tilde{T}_{a}$ is the nondimensional activation energy $(\tilde{E})$, this more explicit representation will not be needed in the stability analysis that follows. Instead, our (linear) stability results may be expressed in terms of the pressure and temperature sensitivities, defined as

$$
\begin{aligned}
& A_{p}=\partial A /\left.\partial p\right|_{\Theta=1, p=0}, \\
& A_{\Theta}=\partial A /\left.\partial \Theta\right|_{\Theta=1, p=0}=N\left(1-\sigma_{u}\right)+\hat{A}_{\Theta},
\end{aligned}
$$

respectively. However, we note that if the nondimensional activation energy is large, then the last of Eqs. (17) implies that $A_{\Theta}$ would likely be larger in magnitude than $A_{p}$, a fact that will play a role in the relative scalings introduced for $A_{p}$ and $A_{\Theta}$ below.

A nontrivial basic solution to the above problem, corresponding to the special case of a steady, planar deflagration, is given by

$$
\begin{gathered}
\Phi_{s}^{0}=-t, \quad p^{0}(z)= \begin{cases}-F r^{-1} z+\rho^{-1}-1, & z<0 \\
-\rho F r^{-1} z, & z>0,\end{cases} \\
\mathbf{v}^{0}=\left(0,0, v^{0}\right), \quad v^{0}= \begin{cases}0, & z<0 \\
\rho^{-1}-1, & z>0,\end{cases} \\
\Theta^{0}(z)= \begin{cases}e^{z}, & z<0 \\
1, & z>0 .\end{cases}
\end{gathered}
$$


The remainder of the paper is devoted to a linear stability analysis of this solution.

\section{The Complete Linear Stability Problem}

Before proceeding with any further approximations, we determine in a straightforward fashion the linear stability problem and the corresponding dispersion relation (in the form of a system of algebraic equations) that govern the behavior of harmonic perturbations about the basic solution (18). In particular, in terms of the perturbation quantities $\phi_{s}(x, y, t)=\Phi_{s}(x, y, z, t)-\Phi_{s}^{0}(t), \mathrm{u}(x, y, z, t)=$ $\mathrm{v}(x, y, z, t)-\mathbf{v}^{0}(z), \zeta(x, y, z, t)=p(x, y, z, t)-p^{0}(z)$ and $\theta(x, y, z, t)=\Theta-\Theta^{0}(z)-\phi_{s} d \Theta^{0} / d z$, the problem obtained when Eqs. (1) - (3), (6) - (12) are linearized about the basic solution (18) is given by

$$
\frac{\partial u_{1}}{\partial x}+\frac{\partial u_{2}}{\partial y}+\frac{\partial u_{3}}{\partial z}=0, \quad z \neq 0,
$$

$$
\begin{aligned}
& \left\{\begin{array}{l}
1 \\
\rho
\end{array}\right\} \frac{\partial \mathbf{u}}{\partial t}+\frac{\partial \mathbf{u}}{\partial z}= \\
& -\left(\frac{\partial \zeta}{\partial x}+\left\{\begin{array}{l}
1 \\
\rho
\end{array}\right\} F r^{-1} \frac{\partial \phi_{s}}{\partial x}, \frac{\partial \zeta}{\partial y}+\left\{\begin{array}{l}
1 \\
\rho
\end{array}\right\} F r^{-1} \frac{\partial \phi_{s}}{\partial y}, \frac{\partial \zeta}{\partial z}\right) \\
& +\left\{\begin{array}{c}
P r_{l} \\
\rho \lambda P r_{g}
\end{array}\right\}\left(\frac{\partial^{2} \mathbf{u}}{\partial x^{2}}+\frac{\partial^{2} \mathbf{u}}{\partial y^{2}}+\frac{\partial^{2} \mathbf{u}}{\partial z^{2}}\right), \quad z \lesseqgtr 0,
\end{aligned}
$$

$$
\begin{aligned}
& \left\{\begin{array}{l}
1 \\
\rho
\end{array}\right\} \frac{\partial \theta}{\partial t}+\frac{\partial \theta}{\partial z}=\left\{\begin{array}{c}
-u_{3} e^{z} \\
0
\end{array}\right\} \\
& +\left\{\begin{array}{c}
1 \\
\rho \lambda
\end{array}\right\}\left(\frac{\partial^{2} \theta}{\partial x^{2}}+\frac{\partial^{2} \theta}{\partial y^{2}}+\frac{\partial^{2} \theta}{\partial z^{2}}\right), \quad z>0, \\
& \mathbf{u}=0, \theta=0 \text { at } z=-\infty \text {, } \\
& \theta=0 \text { at } z=+\infty,\left.\quad \theta\right|_{z=0^{+}}-\left.\theta\right|_{z=0^{-}}=\phi_{s} \text {, } \\
& \left.u_{1}\right|_{z=0^{-}}-\left.u_{1}\right|_{z=0^{+}}=\left(\rho^{-1}-1\right) \frac{\partial \phi_{s}}{\partial x}, \\
& \left.u_{2}\right|_{z=0^{-}}-\left.u_{2}\right|_{z=0^{+}}=\left(\rho^{-1}-1\right) \frac{\partial \phi_{s}}{\partial y}, \\
& \left.u_{3}\right|_{z=0^{-}}-\left.\rho u_{3}\right|_{z=0^{+}}=(1-\rho) \frac{\partial \phi_{s}}{\partial t}, \\
& \left.u_{3}\right|_{z=0^{-}}-\frac{\partial \phi_{s}}{\partial t}=\left.A_{p} \zeta\right|_{z=0^{+}}+\left.A_{\ominus} \theta\right|_{z=0^{+}}, \\
& \left.\zeta\right|_{z=0^{-}}-\left.\zeta\right|_{z=0^{+}}=\left.(2-\rho) u_{3}\right|_{z=0^{+}}-\left.u_{3}\right|_{z=0^{-}} \\
& +\left.2 \operatorname{Pr}_{l} \frac{\partial u_{3}}{\partial z}\right|_{z=0-}-\left.2 \rho \lambda P r_{g} \frac{\partial u_{3}}{\partial z}\right|_{z=0^{+}} \\
& -(1-\rho) \frac{\partial \phi_{s}}{\partial t}-\gamma\left(\frac{\partial^{2} \phi_{s}}{\partial x^{2}}+\frac{\partial^{2} \phi_{s}}{\partial y^{2}}\right),
\end{aligned}
$$

$$
\begin{aligned}
& \rho \lambda \operatorname{Pr}_{g}\left(\left.\frac{\partial u_{1}}{\partial z}\right|_{z=0^{+}}+\left.\frac{\partial u_{3}}{\partial x}\right|_{z=0^{+}}\right) \\
& -\operatorname{Pr} l\left(\left.\frac{\partial u_{1}}{\partial z}\right|_{z=0^{-}}+\left.\frac{\partial u_{3}}{\partial x}\right|_{z=0^{-}}\right) \\
& =\left(\rho^{-1}-1\right) \frac{\partial \phi_{s}}{\partial x}+\left.u_{1}\right|_{z=0^{+}}-\left.u_{1}\right|_{z=0^{-}} \text {, } \\
& \rho \lambda \operatorname{Pr}_{g}\left(\left.\frac{\partial u_{2}}{\partial z}\right|_{z=0^{+}}+\left.\frac{\partial u_{3}}{\partial y}\right|_{z=0^{+}}\right) \\
& -\operatorname{Pr}\left(\left.\frac{\partial u_{2}}{\partial z}\right|_{z=0^{-}}+\left.\frac{\partial u_{3}}{\partial y}\right|_{z=0^{-}}\right) \\
& =\left(\rho^{-1}-1\right) \frac{\partial \phi_{s}}{\partial y}+\left.u_{2}\right|_{z=0^{+}}-\left.u_{2}\right|_{z=0^{-}}, \\
& \left.\operatorname{co\lambda } \frac{\partial \theta}{\partial z}\right|_{z=0^{+}}-\left.\frac{\partial \theta}{\partial z}\right|_{z=0^{-}}-\left.c \theta\right|_{z=0^{+}}+\left.\theta\right|_{z=0^{-}} \\
& =\left.\hat{c} \rho u_{3}\right|_{z=0^{+}}-\left.(1+\hat{c}) u_{3}\right|_{z=0^{-}}+[1+\hat{c}(1-\rho)] \frac{\partial \phi_{s}}{\partial t},
\end{aligned}
$$

where $\hat{c} \equiv c\left(1-\sigma_{u}\right)^{-1}$.

Nontrivial harmonic solutions for $\phi_{s}, \mathbf{u}$ and $\zeta$, proportional to $e^{i \omega t+i k_{1} x+i k_{2} y}$, that satisfy Eqs. (19) - (21) and the boundary/boundedness conditions at $z= \pm \infty$ are given by

$$
\phi_{s}=e^{i \omega t+i k_{1} x+i k_{2} y},
$$

and

$$
\begin{gathered}
\zeta=e^{i \omega t+i k_{1} x+i k_{2} y}\left\{\begin{array}{l}
b_{1} e^{k z}-F r^{-1} \\
b_{2} e^{-k z}-\rho F r^{-1},
\end{array}\right. \\
u_{1}=e^{i \omega t+i k_{1} x+i k_{2} y}\left\{\begin{array}{l}
b_{3} e^{q z}-i k_{1}(i \omega+k)^{-1} b_{1} e^{k z} \\
b_{4} e^{r z}-i k_{1}(i \omega \rho-k)^{-1} b_{2} e^{-k z}
\end{array}\right. \\
u_{2}=e^{i \omega t+i k_{1} x+i k_{2} y}\left\{\begin{array}{l}
b_{5} e^{q z}-i k_{2}(i \omega+k)^{-1} b_{1} e^{k z} \\
b_{6} e^{r z}-i k_{2}(i \omega \rho-k)^{-1} b_{2} e^{-k z}
\end{array}\right. \\
u_{3}=e^{i \omega t+i k_{1} x+i k_{2} y}\left\{\begin{array}{l}
b_{7} e^{q z}-k(i \omega+k)^{-1} b_{1} e^{k z} \\
b_{8} e^{r z}+k(i \omega \rho-k)^{-1} b_{2} e^{-k z}
\end{array}\right. \\
\theta=e^{i \omega t+i k_{1} x+i k_{2} y} \\
\cdot\left\{\begin{array}{l}
b_{9} e^{p z}-\left[i \omega+k^{2}-q(q+1)\right]^{-1} b_{7} e^{(q+1) z} \\
b_{10} e^{s z}, \quad+k\left[(i \omega)^{2}-k^{2}\right]^{-1} b_{1} e^{(k+1) z}
\end{array}\right.
\end{gathered}
$$

for $z \lessgtr 0$, where we have normalized the above solution by setting the coefficient of the harmonic dependence of $\phi_{s}$ to unity. Here, the signs of $k_{1}$ and $k_{2}$ may be either positive or negative and we have employed the definition $k=\sqrt{k_{1}^{2}+k_{2}^{2}}$, and $p, q, r$ and $s$ are defined as

$$
2 p=1+\sqrt{1+4\left(i \omega+k^{2}\right)}
$$




$$
\begin{gathered}
2 P r_{l} q=1+\sqrt{1+4 P r_{l}\left(i \omega+P r_{l} k^{2}\right)}, \\
2 \mu P r_{l} r=1-\sqrt{1+4 \mu P r_{l}\left(i \omega \rho+\mu P r_{l} k^{2}\right)}, \\
2 \rho \lambda s=1-\sqrt{1+4 \rho^{2} \lambda\left(i \omega+\lambda k^{2}\right)},
\end{gathered}
$$

where we have used the fact, noted below Eq. (5), that $\rho \lambda P r_{g}=\mu P r_{l}$.

Substituting this solution into the interface conditions (23) - (30) and using Eq. (19) for $z>0$ yields eleven conditions for the ten coefficients $b_{1}-b_{10}$ and the complex frequency (dispersion relation) $i \omega(k)$. In particular, these conditions are given by

$$
\begin{aligned}
& i k_{1} b_{3}+i k_{2} b_{5}+q b_{7}=0 \\
& i k_{1} b_{4}+i k_{2} b_{6}+r b_{8}=0 \text {, } \\
& b_{3}-\frac{i k_{1}}{i \omega+k} b_{1}-b_{4}+\frac{i k_{1}}{i \omega \rho-k} b_{2}=\left(\frac{1}{\rho}-1\right) i k_{1} \text {, } \\
& b_{5}-\frac{i k_{2}}{i \omega+k} b_{1}-b_{6}+\frac{i k_{2}}{i \omega \rho-k} b_{2}=\left(\frac{1}{\rho}-1\right) i k_{2} \text {, } \\
& b_{7}-\frac{k}{i \omega+k} b_{1}-\rho b_{8}-\frac{\rho k}{i \omega \rho-k} b_{2}=(1-\rho) i \omega, \\
& b_{7}-\frac{k}{i \omega+k} b_{1}-A_{p} b_{2}-A_{\Theta} b_{10}=i \omega-\rho F r^{-1} A_{p} \text {, } \\
& {\left[1+\frac{k}{i \omega+k}\left(2 k P r_{l}-1\right)\right] b_{1}} \\
& -\left[1+\frac{k}{i \omega \rho-k}\left(2 k \mu P r_{l}+2-\rho\right)\right] b_{2} \\
& +\left(1-2 P r_{l} q\right) b_{7}-\left(2-\rho-2 \mu P r_{l} r\right) b_{8} \\
& =(1-\rho)\left(F r^{-1}-i \omega\right)+\gamma k^{2} \text {, } \\
& \left(\mu P r_{l} \tau-1\right) b_{4}+\left(2 k \mu P r_{l}+1\right) \frac{i k_{1}}{i \omega \rho-k} b_{2} \\
& +i k_{1} \mu P r_{l} b_{8}+\left(1-P r_{l} q\right) b_{3} \\
& +\left(2 k P r_{l}-1\right) \frac{i k_{1}}{i \omega+k} b_{1}-i k_{1} P r_{l} b_{7} \\
& =\left(\frac{1}{\rho}-1\right) i k_{1}, \\
& \left(\mu P r_{l} r-1\right) b_{6}+\left(2 k \mu P r_{l}+1\right) \frac{i k_{2}}{i \omega \rho-k} b_{2} \\
& +i k_{2} \mu P r_{l} b_{8}+\left(1-P r_{l} q\right) b_{5} \\
& +\left(2 k P r_{l}-1\right) \frac{i k_{2}}{i \omega+k} b_{1}-i k_{2} P r_{l} b_{7} \\
& =\left(\frac{1}{\rho}-1\right) i k_{2} \text {, }
\end{aligned}
$$

$b_{10}-b_{9}+\left[i \omega+k^{2}-q(q+1)\right]^{-1} b_{7}-k\left[(i \omega)^{2}-k^{2}\right]^{-1} b_{1}=1$,

$$
\begin{aligned}
& (1-c+c \rho \lambda s) b_{10}-p b_{9}-\hat{c} \rho b_{8} \\
& +\left[\frac{q+1}{i \omega+k^{2}-q(q+1)}+1+\hat{c}\right] b_{7} \\
& -\frac{k}{i \omega+k}\left[\frac{k+1}{i \omega-k}+1+\hat{c}\right] b_{1}-\frac{\hat{c} \rho k}{i \omega \rho-k} b_{2} \\
& =1+i \omega[1+\hat{c}(1-\rho)] .
\end{aligned}
$$

While the above problem is linear in the coefficients $b_{1}-b_{8}$, the relationship for $i \omega$ is highly nonlinear. Accordingly, we seek asymptotic solutions for the neutral stability boundaries in the limit that the gas-to-liquid density and viscosity ratios $\rho$ and $\mu$ are small, as is $\mathrm{Fr}^{-1}$ in the case of reduced gravity. We shall also introduce appropriate scalings for the sensitivity parameters $A_{p}$ and $A_{\Theta}$, where the limiting results for $A_{\ominus}=0$ were derived previously $[13,14]$. In particular, we introduce a bookkeeping parameter $\epsilon \ll 1$ and consider the realistic parameter regime

$$
\rho=\rho^{*} \epsilon, \quad \mu=\mu^{*} \epsilon, \quad F r^{-1}=\left\{\begin{array}{l}
g \sim O(1) \\
g^{*} \epsilon \sim O(\epsilon),
\end{array}\right.
$$

where the the upper (lower) choice for $\mathrm{Fr}^{-1}$ corresponds to normal (reduced) gravity. We note that based on characteristic densities for liquid propellants and product gases at elevated temperatures, a realistic value for $\epsilon$ is on the order of $10^{-3}$ or $10^{-4}$. In this parameter regime, it was seen that the appropriate scaling for $A_{p}$ to describe the neutral stability region was $A_{p}=A_{p}^{*} \epsilon$, whereas the appropriate scale that describes the fully-developed effects of thermal coupling turns out to be $A_{\Theta}=A_{\Theta}^{*} \epsilon^{1 / 4}$, as shown in Section 6 below. Thus, we observe that $A_{\Theta} / A_{p} \sim O\left(\epsilon^{-3 / 4}\right) \geq 175$, which is at least several times as large as typical values of the nondimensional activation energy $N$, but still consistent with the last of Eqs. (17). However, as shown in Section 7 , the first significant effects of thermal coupling are in fact felt on an intermediate scale $A_{\Theta} \sim \mathrm{O}\left(\epsilon^{1 / 2}\right)$, in which case $A_{\Theta} / A_{p} \sim \mathrm{O}\left(\epsilon^{-1 / 2}\right) \geq 30$, which is a quite typical value for $N$. Thus, the parameter regime of interest for describing modifications to the hydrodynamic stability boundaries arising from temperature-sensitivity effects coincides with realistic values of the sensitivity coefficients.

\section{The Inviscid Limit}

Although both liquid and gas-phase viscous effects were shown to be generally comparable to those due to surface tension when thermal coupling was neglected, ${ }^{13,14}$ the qualitative nature of the cellular boundary remains preserved in the zero-viscosity 
limit, as does the existence of the pulsating boundary. Accordingly, we shall, for simplicity, mainly consider the effects of thermal sensitivity on hydrodynamic stability in the inviscid limit, reserving the detailed consideration of thermal coupling in the algebraically more involved viscous case for a future study. ${ }^{19}$ In particular, before introducing the scalings indicated above, we first set $P r_{l}=P r_{g}=0$. Consequently, since $q \rightarrow \infty$ according to Eq. (38), we set $b_{3}=b_{5}=b_{7}=0$ in Eqs. (33) - (35), and observing that Eqs. (48) - (49) then reduce to the no-slip conditions $(43)-(44)$, we thus eliminate Eqs. (41), (48) and (49) from the above set. Using the fact that $r \rightarrow-i \omega \rho$, the solution for the remaining coefficients in terms of $i \omega$ is given by

$$
\begin{gathered}
b_{8}=\frac{k}{2 \rho(i \omega \rho-k)(i \omega \rho+k)} \\
\quad\left\{(1-\rho)^{2}\left[(i \omega)^{2} \rho+k^{2}\right]\right. \\
\left.+\rho\left(1-\rho^{2}\right) F r^{-1} k+\rho(1+\rho) \gamma k^{3}\right\}, \\
b_{1}=-\frac{i \omega+k}{(1+\rho) k^{2}}\left[(i \omega+k)(1-\rho) k-\rho(i \omega \rho-k) b_{8}\right], \\
b_{2}=-\frac{i \omega \rho-k}{\rho(1+\rho) k^{2}}\left[(i \omega \rho-k)(1-\rho) k+\rho^{2}(i \omega+k) b_{8}\right], \\
b_{4}=\frac{\omega \rho k_{1}}{k^{2}} b_{8}, \quad b_{6}=\frac{\omega \rho k_{2}}{k^{2}} b_{8}, \\
A_{\Theta} b_{10}=A_{p}\left[\rho F r^{-1}+\frac{(i \omega \rho-k)^{2}(1-\rho)}{\rho(1+\rho) k}\right. \\
\left.+\frac{\rho(i \omega \rho-k)(i \omega+k)}{(1+\rho) k^{2}} b_{8}\right] \\
\quad+\frac{(1-\rho) k-2 i \omega \rho}{1+\rho}-\frac{\rho(i \omega \rho-k)}{(1+\rho) k} b_{8}, \\
b_{9}=b_{10}-1+\frac{(i \omega+k)(1-\rho) k-\rho(i \omega \rho-k) b_{8}}{(1+\rho)(i \omega-k) k} .
\end{gathered}
$$

Substitution of these results into Eq. (51) then gives a single implicit equation for $i \omega$ given by

$$
\begin{gathered}
{[c(\rho \lambda s-1)+1-p] b_{10}} \\
-\frac{1-\rho}{1+\rho}\left[\frac{i \omega+k}{i \omega-k}(p-k-1)-(1+\hat{c}+\hat{c} \rho) i \omega-k\right] \\
+\frac{\rho}{(1+\rho) k}\left[\frac{i \omega \rho-k}{i \omega-k}(p-k-1)-(i \omega \rho-k)\right] b_{8} \\
=1-p+i \omega[1+\hat{c}(1-\rho)],
\end{gathered}
$$

where $b_{8}(i \omega)$ and $b_{10}(i \omega)$ are given explicitly by Eqs. (53) and (57), provided $A_{\ominus} \neq 0$. Equation (59) is thus the dispersion relation that determines the neutral stability boundaries in that case. In the limit $A_{\Theta} \rightarrow 0$, the dispersion relation is given by setting the right-hand side of Eq. (57) to zero, in which case Eqs. (58) and (59), which are then decoupled from Eqs. (53) - (57), determine $b_{9}$ and $b_{10}$. Alternatively, we may solve Eq. (59) for $b_{10}(i \omega)$ and substitute this result into Eq. (57), which then becomes the dispersion relation for arbitrary $A_{\ominus}$. We remark that the special case $A_{\Theta}=0$ yields an analytically tractable solution for the neutral stability boundaries, ${ }^{12}$ but since all other results to be presented are obtained for the parameter regime defined by Eqs. (52), we shall restrict ourselves to this regime for this limiting case as well.

\section{Hydrodynamic Stability Boundaries in the Limit $A_{\Theta}=0$}

In the limit $A_{\Theta}=0$, the results obtained from the dispersion relation (57) in the parameter regime (52) are as follows. ${ }^{13}$ First (see Figure 1), the pulsating hydrodynamic stability boundary is given, to leading order in $\epsilon$, by $A_{p}^{*}=-\rho^{*}$ for all disturbance wavenumbers [the exact relation is given by $A_{p}^{*}=$ $\left.-\rho^{*} /\left(1-\epsilon \rho^{*}\right)\right]$, with instability occurring below this critical value. For the cellular stability boundary, the corresponding leading-order expression depends on the magnitude of the wavenumber $k$. In particular, there are three wavenumber scales to be considered; the $\mathrm{O}(1)$, or outer, scale $k$, a far outer scale $k_{f}=k \epsilon$, and an inner scale defined by either $k_{i}=$ $k / \epsilon$ if $F r^{-1} \sim O(1)$ or $k_{i}=k / \epsilon^{2}$ if $F r^{-1} \sim O(\epsilon)$. In each of these regions, the cellular stability boundary, on which the complex frequency $i \omega$ is identically zero, is given by

$$
A_{p}^{*} \sim A_{p}^{*(o)} \sim-\frac{1}{2} \rho^{*}
$$

$$
A_{p}^{*} \sim A_{p}^{*(f)} \sim \frac{1}{2} \rho^{*}\left(\rho^{*} \gamma k_{f}-1\right)
$$

and

$$
A_{p}^{*} \sim A_{p}^{*(i)} \sim \begin{cases}\rho^{*}\left(\rho^{*} g-k_{i}\right) / 2 k_{i}, & F r^{-1} \sim \mathrm{O}(1) \\ \rho^{*}\left(\rho^{*} g^{*}-k_{i}\right) / 2 k_{i}, & F r^{-1} \sim \mathrm{O}(\epsilon)\end{cases}
$$

respectively. Matching the above solutions to one another, a uniformly valid composite expansion, denoted by $A_{p}^{*(c)}(k)$, can be constructed in the region $A_{p}^{*}<0$ (the basic solution can be shown to be un- 
stable for $\left.A_{p}^{*}>0\right)$. In particular,

$$
\begin{aligned}
A_{p}^{*(c)} \sim & A_{p}^{*(i)}+A_{p}^{*(o)}+A_{p}^{*(f)} \\
& -\lim _{k_{i} \rightarrow \infty} A_{p}^{*(i)}-\lim _{k, \rightarrow 0} A_{p}^{*(f)} \\
\sim- & \frac{1}{2} \rho^{*}+\frac{1}{2} \epsilon \rho^{* 2} \gamma k \\
& + \begin{cases}\epsilon \rho^{* 2} g / 2 k, & F r^{-1} \sim \mathrm{O}(1) \\
\epsilon^{2} \rho^{* 2} g^{*} / 2 k, & F r^{-1} \sim \mathrm{O}(\epsilon)\end{cases}
\end{aligned}
$$

where the definitions of $k_{i}$ and $k_{f}$ have been used to express the final result in terms of $k$. We note that this asymptotic result, exhibited in Figure 1, is far simpler than the exact result, ${ }^{12}$ which, in our present notation, is given in the region $A_{p}^{*}<0$ by

$$
\begin{aligned}
& A_{p}^{*} / \rho^{*}= \\
& \frac{\rho^{*} \epsilon\left(1-\rho^{*} \epsilon\right) F r^{-1}+\rho^{*} \epsilon \gamma k^{2}-\left(1-\rho^{*} \epsilon\right) k}{\rho^{* 2} \epsilon^{2}\left(3-\rho^{*} \epsilon\right) F r^{-1}+\rho^{* 2} \epsilon^{2} \gamma k^{2}+\left(1-\rho^{*} \epsilon\right)\left(2-\rho^{*} \epsilon\right) \bar{k}}
\end{aligned}
$$

It is thus easily seen from Figure 1 that there is a stable region between the pulsating and cellular stability boundaries for $A_{\Theta}=0$, and this result is preserved (in fact, enhanced) when viscous effects, which play a stabilizing role with respect to both boundaries for sufficiently large wavenumbers, are included in the analysis. ${ }^{13,14}$ We observe that for the Landau (cellular) form of hydrodynamic instability, gravity and surface tension are stabilizing with respect to sufficiently small and sufficiently large wavenumber disturbances, respectively. The essential difference, as illustrated in Figure 1, between the normal and reduced gravity limits in the realistic parameter regime considered here is that in the latter instance, gravity is only capable of stabitizing disturbances whose wavenumbers are very small, $O\left(\epsilon^{2}\right)$, whereas in the former case, gravity is sufficiently strong to stabilize disturbances whose wavenumbers are $O(\epsilon)$. As a consequence, hydrodynamic instability becomes a long-wave instability phenomenon in the reduced gravity regime considered here, since, from Eq. (63), the most unstable wavenumbers are $O(\sqrt{\epsilon})$, rather than $O(1)$, in that case. We remark that although positive values of the pressure sensitivity $A_{p}$ are hydrodynamically unstable, zero and negative values of $A_{p}$ over certain pressure ranges are, in fact, characteristic of the so-called "plateau" and "mesa" types of solid propellants, ${ }^{17}$ as well as for the HAN-based liquid propellants mentioned above. ${ }^{15}$

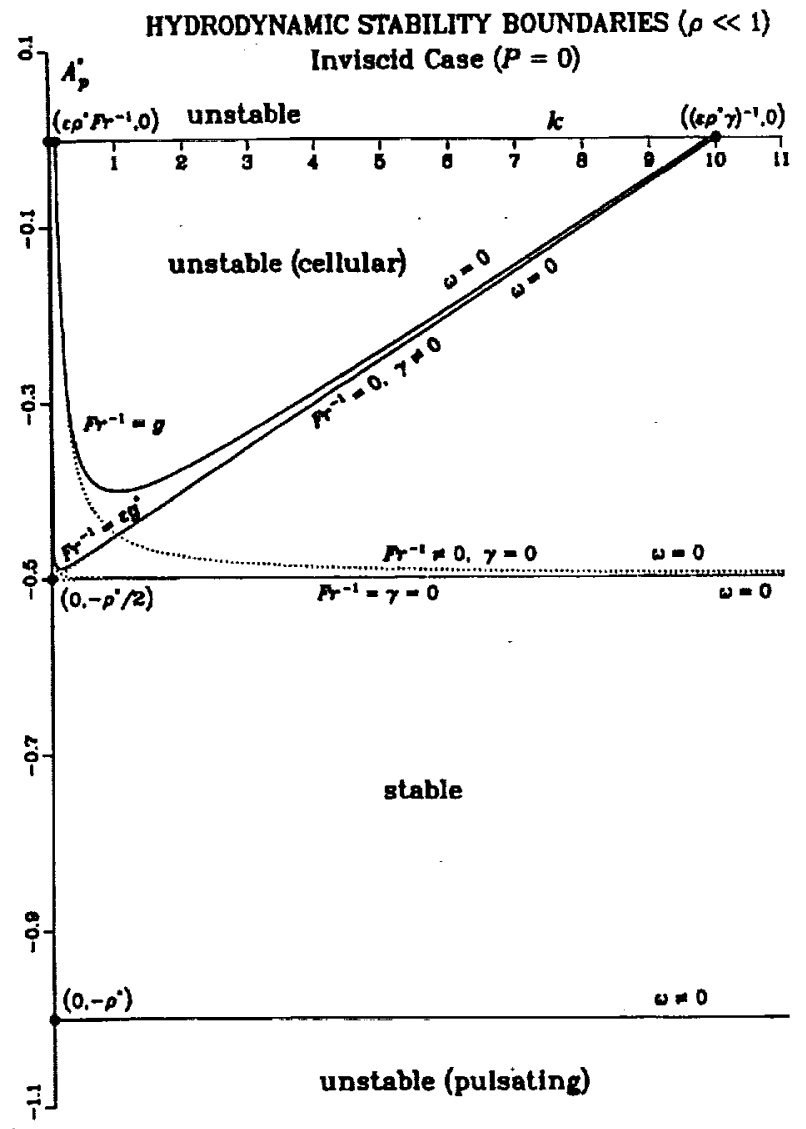

Figure 1. Inviscid hydrodynamic stability boundaries in the limit of zero thermal coupling $\left(A_{\ominus}=0\right)$.

\section{Hydrodynamic Stability Boundaries for $A_{\Theta}>0$}

Retaining the parameter scalings introduced in Section 3, we first consider the $O(1)$ wavenumber regime. For the case $A_{\Theta}=0$ considered previously, it turns out ${ }^{14}$ that the appropriate expansion of the dispersion relation $i \omega(k)$ is of the form

$$
i \omega \sim \epsilon^{-1 / 2}\left(i \omega_{0}+\epsilon^{1 / 4} i \omega_{1}+\epsilon^{1 / 2} i \omega_{2}+\cdots\right),
$$

where the leading-order term was suggested by the explicit results available for the inviscid case, and the expansion in quarter powers of $\epsilon$ is suggested by the leading-order term in the resulting expansion for $p$ given below. We now introduce the scaling $A_{\Theta}=\epsilon^{n} A_{\Theta}^{*}$, where the value of $n \geq 0$ is to be determined so as to give a nontrivial modification in the dispersion relation at the appropriate order, corresponding to the first effects of a nonzero temperature sensitivity on the neutral stability boundary for $\mathrm{O}(1)$ wavenumbers. 
Substituting Eq. (65) and the various scalings into Eqs. (37), (40) and (53), expanded forms for $p$, $s$ and $b_{8}$ are obtained as

$$
\begin{gathered}
p \sim p_{0} \epsilon^{-1 / 4}+p_{1}+p_{2} \epsilon^{1 / 4}+\cdots, \quad s \sim \mathrm{O}\left(\epsilon^{1 / 2}\right), \quad(66) \\
b_{8} \sim-\frac{\left(i \omega_{0}\right)^{2} \rho^{*}+k^{2}}{2 \rho^{*} k} \epsilon^{-1}-\frac{1}{k}\left(i \omega_{0}\right)\left(i \omega_{1}\right) \epsilon^{-3 / 4}, \\
-\frac{1}{2 k}\left[\left(i \omega_{1}^{2}\right)+2\left(i \omega_{0}\right)\left(i \omega_{2}\right)\right] \epsilon^{-1 / 2}+\cdots,
\end{gathered}
$$

where

$$
\begin{aligned}
& p_{0}=\left(i \omega_{0}\right)^{1 / 2}, \quad p_{1}=\frac{1}{2}\left[i \omega_{1} /\left(i \omega_{0}\right)^{1 / 2}+1\right], \\
& p_{2}=\frac{1}{8}\left(i \omega_{0}\right)^{-1 / 2}\left[1+4 k^{2}+4 i \omega_{2}-\left(i \omega_{1}\right)^{2} / i \omega_{0}\right] .
\end{aligned}
$$

Consequently, from Eqs. (57) and (67), $b_{10}$ has the expanded form

$$
\begin{aligned}
& b_{10} \sim \\
& \epsilon^{-n} \frac{A_{p}^{*}}{A_{\Theta}^{*}}\left\{\frac{k}{\rho^{*}}+\frac{i \omega_{0}}{2 k^{2}}\left[\left(i \omega_{0}\right)^{2} \rho^{*}-3 k^{2}\right] \epsilon^{1 / 2}+\cdots\right\} \\
& +\frac{\epsilon^{-n}}{A_{\ominus}^{*}}\left\{-\frac{1}{2 k}\left[\left(i \omega_{0}\right)^{2} \rho^{*}-k^{2}\right]-\frac{\rho^{*}}{k}\left(i \omega_{0}\right)\left(i \omega_{1}\right) \epsilon^{1 / 4}\right. \\
& +\frac{\rho^{*}}{2 k}\left[\frac{i \omega_{0}}{k}\left\{\left(i \omega_{0}\right)^{2} \rho^{*}-3 k^{2}\right\}\right. \\
& \left.\left.-\left(i \omega_{1}\right)^{2}-2\left(i \omega_{0}\right)\left(i \omega_{2}\right)\right] \epsilon^{1 / 2}+\cdots\right\} \\
& \sim \epsilon^{-n}\left[b_{10}^{(0)}+b_{10}^{(1)} \epsilon^{1 / 4}+b_{10}^{(2)} \epsilon^{1 / 2}+\cdots\right]
\end{aligned}
$$

which, when substituted into the dispersion relation (59) along with the other expansions deduced thus far, yields a sequence of equations for the determination of the coefficients in the expansion (65). Thus, at leading order, $\mathrm{O}\left(\epsilon^{-n-1 / 4}\right)$, Eq. (59) yields $b_{10}^{(0)}=0$, which, from the implied definition of $b_{10}^{(0)}$ in Eq. (69) above, gives

$$
A_{p}^{*} \frac{k}{\rho^{*}}-\frac{1}{2 k}\left[\left(i \omega_{0}\right)^{2} \rho^{*}-k^{2}\right]=0,
$$

or

$$
\left(i \omega_{0}\right)^{2}=\left(\frac{k}{\rho^{*}}\right)^{2}\left(2 A_{p}^{*}+\rho^{*}\right) .
$$

Hence, $i \omega_{0}$ is identically zero for $A_{p}^{*}=-\rho^{*} / 2$, which corresponds to the leading-order hydrodynamic cellular boundary (60) in the $O(1)$ wavenumber regime when $A_{\Theta}^{*}=0$. Values of $A_{p}^{*}>-\rho^{*} / 2$ imply cellular instability, since in that case there exists, according to Eq. (70b), a root of the dispersion relation such that $\operatorname{Re}\left\{i \omega_{0}\right\}>0, \mathcal{I} m\left\{i \omega_{0}\right\}=0$. On the other hand, the stability of the region $A_{p}^{*}<$ $-\rho^{*} / 2$ is still indeterminate, since Eq. (70b) indicates that $\operatorname{Re}\left\{i \omega_{0}\right\}=0$ there. Hence, the next nontrivial term in the expansion of $i \omega$ is required to determine whether this region is stable or unstable, although the fact that $\operatorname{Im}\left\{i \omega_{0}\right\} \neq 0$ implies that disturbances have a pulsating character for values of $A_{p}^{*}$ below the cellular stability boundary.

At the next order, $O\left(\epsilon^{-n}\right)$, in the analysis of the dispersion relation, the equation $b_{10}^{(1)}=0$ is obtained, whence the definition of $b_{10}^{(1)}$ in Eq. (69) implies that $i \omega_{1}=0$. Proceeding with the next higher order equation obtained at $O\left(\epsilon^{-n+1 / 4}\right)$ from the expanded version of Eq. (59), it can be seen that terms other than those proportional to $A_{\Theta}^{*}{ }^{-1}$ will appear provided the choice $n=1 / 4$ is made. In that case, collecting terms of $O\left(\epsilon^{0}\right)$ and using the previous results $b_{10}^{(0)}=b_{10}^{(1)}=0$ leads to the equation

$$
-p_{0} b_{10}^{(2)}+2 k-\frac{1}{2 k}\left[\left(i \omega_{0}\right)^{2} \rho^{*}+k^{2}\right]=0
$$

which, upon using the definitions of $p_{0}$ and $b_{10}^{(2)}$, may be solved for $i \omega_{2}$ as

$$
\begin{aligned}
& i \omega_{2}= \\
& \quad \frac{1}{2 \rho^{*} k}\left[\left(i \omega_{0}\right)^{2} \rho^{*}-3 k^{2}\right]\left[A_{p}^{*}+\rho^{*}+k A_{\Theta}^{*}\left(i \omega_{0}\right)^{-3 / 2}\right] \\
& =\frac{k}{\rho^{* 2}}\left(A_{p}^{*}-\rho^{*}\right) \\
& \quad \cdot\left[A_{p}^{*}+\rho^{*}+\rho^{* 3 / 2} k^{-1 / 2} A_{\Theta}^{*}\left(2 A_{p}^{*}+\rho^{*}\right)^{-3 / 4}\right],
\end{aligned}
$$

where the expression (70b) for $i \omega_{0}$ has been used to obtain the final equality. It is readily seen that for $A_{\ominus}^{*}=0, \operatorname{Re}\left\{i \omega_{2}\right\}=0$ for $A_{p}^{*}=-\rho^{*}<-\rho^{*} / 2$. -That is, as described in the previous section, there exists, since $\operatorname{Im}\{i \omega\} \neq 0$, a pulsating neutral stability boundary that lies below the cellular boundary such that the region between the two, namely $-\rho^{*} / 2<A_{p}^{*}<-\rho^{*}$, is stable $\left[\operatorname{Re}\left\{i \omega_{2}\right\}<0\right]$, and the region below the pulsating boundary is unstable $\left[\mathcal{R} e\left\{i \omega_{2}\right\}>0\right]$.

For $A_{\ominus}^{*}>0$, the pulsating boundary is modified. In particular, in the region $A_{p}^{*}<-\rho^{*} / 2$ below the cellular boundary, the principal value of the complex factor in Eq. (71b) may be expressed as $\left(2 A_{p}^{*}+\rho^{*}\right)^{-3 / 4}=\left[-\left(2 A_{p}^{*}+\rho^{*}\right)\right]^{-3 / 4} e^{-3 i \pi / 4}$, and thus the neutral stability condition $\operatorname{Re}\left\{i \omega_{2}\right\}=0$ is given by

$$
A_{p}^{*}+\rho^{*}-\frac{1}{2} \sqrt{2} \rho^{* 3 / 2} k^{-1 / 2} A_{\Theta}^{*}\left[-\left(2 A_{p}^{*}+\rho^{*}\right)\right]^{-3 / 4}=0 \text {. }
$$


Writing $A_{p}^{*}=-\rho^{*}+\hat{a} \rho^{*} / 2$, where $\hat{a}$ represents the deviation, in units of $\rho^{*} / 2$, in the pulsating stability boundary from its value in the zero-thermalsensitivity limit $A_{\Theta}^{*}=0$, the condition (72) becomes $\hat{a}-\sqrt{2} \rho^{*-1 / 4} k^{-1 / 2} A_{\Theta}^{*}(1-\hat{a})^{-3 / 4}=0$, or equivalently,

$$
\hat{a}^{4}(1-\hat{a})^{3}=4 \rho^{*-1} k^{-2} A_{\ominus}^{* 4} .
$$

Equation (73a) is an implicit expression for the pulsating stability boundary $\hat{a}(k)$, where the value $\hat{a}=$ 1 corresponds to the cellular boundary and $\hat{a}=0$ is the pulsating boundary for $A_{\Theta}^{*}=0$. Alternatively, writing $A_{p}^{*}=\left(-\rho^{*} / 2\right)(1+\hat{b})$, where $\hat{b}=1-\hat{a}$ is the negative deviation, in units of $\rho^{*} / 2$, from the cellular stability boundary $A_{p}^{*}=-\rho^{*} / 2$, Eq. (73a) can be rewritten in terms of $\hat{b}$ as

$$
\hat{b}^{3}(1-\hat{b})^{4}=4 \rho^{*-1} k^{-2} A_{\Theta}^{*},
$$

where $\hat{b}=1(\hat{b}=0)$ corresponds to the pulsating (cellular) boundary in the limit $A_{\Theta}^{*}=0$. A plot of $k(\hat{b})$ for several different values of $A_{\Theta}^{*}$ is shown in Figure 2, which, when rotated $-90^{\circ}$ so that the $k$-axis is horizontal, is readily interpreted in the context of Figure 1 , the lines $A_{p}^{*}=-\rho^{*} / 2$ and $A_{p}^{*}=-\rho^{*}$ in that figure corresponding to $\hat{b}=0$ and $\hat{b}=1$, respectively, in Figure 2.

Referring to Figure 2, it is seen that for $A_{\ominus}^{*}>$ 0 , the pulsating boundary becomes $\mathrm{C}$-shaped (in the rotated frame of reference), the upper branch approaching the cellular boundary $\hat{b}=0$ as $k \rightarrow$ $\infty$, and the lower branch approaching the original $\left(A_{\Theta}^{*}=0\right)$ pulsating boundary $\hat{b}=1$. These same limits are approached for any fixed value of $k$ as $A_{\Theta}^{*} \rightarrow 0$. The portion within the $\mathrm{C}$-shaped curve is the stable region, and thus not only is steady, planar burning intrinsically unstable for sufficiently small wavenumbers, but, for finite values of $k$, any crossing of the C-shaped boundary from the stable to the unstable region corresponds to the onset of a pulsating instability. As $A_{\Theta}^{*}$ increases, the turning point of the C-shaped pulsating boundary, which occurs at $\hat{b}=\hat{b}_{c}=3 / 7$ (i.e., at $A_{p}^{*}=-5 \rho^{*} / 7$ ) corresponding to the critical wavenumber $k=k_{c}=$ $(343 / 72)\left(21 / \rho^{*}\right)^{1 / 2} A_{\Theta}^{* 2} \approx 21.83 A_{\Theta}^{* 2} / \rho^{* 1 / 2}$, shifts to larger values of $k$ as $A_{\ominus}^{*}$ increases. On the other hand, as $A_{\ominus}^{*}$ becomes small, the turning point shifts to small values of $k$ such that $k_{\mathrm{c}}$ eventually leaves the $O(1)$ wavenumber region for which Eqs. (73) are valid. Thus, as $A_{\Theta}^{*}$ becomes small, the original pulsating and cellular boundaries are recovered in the $\mathrm{O}(1)$ wavenumber regime, but as $A_{\Theta}^{*}$ becomes large, the original cellular boundary lies within the unstable region for $\mathrm{O}(1)$ wavenumbers, and the basic solution becomes intrinsically unstable to oscillatory disturbances.*

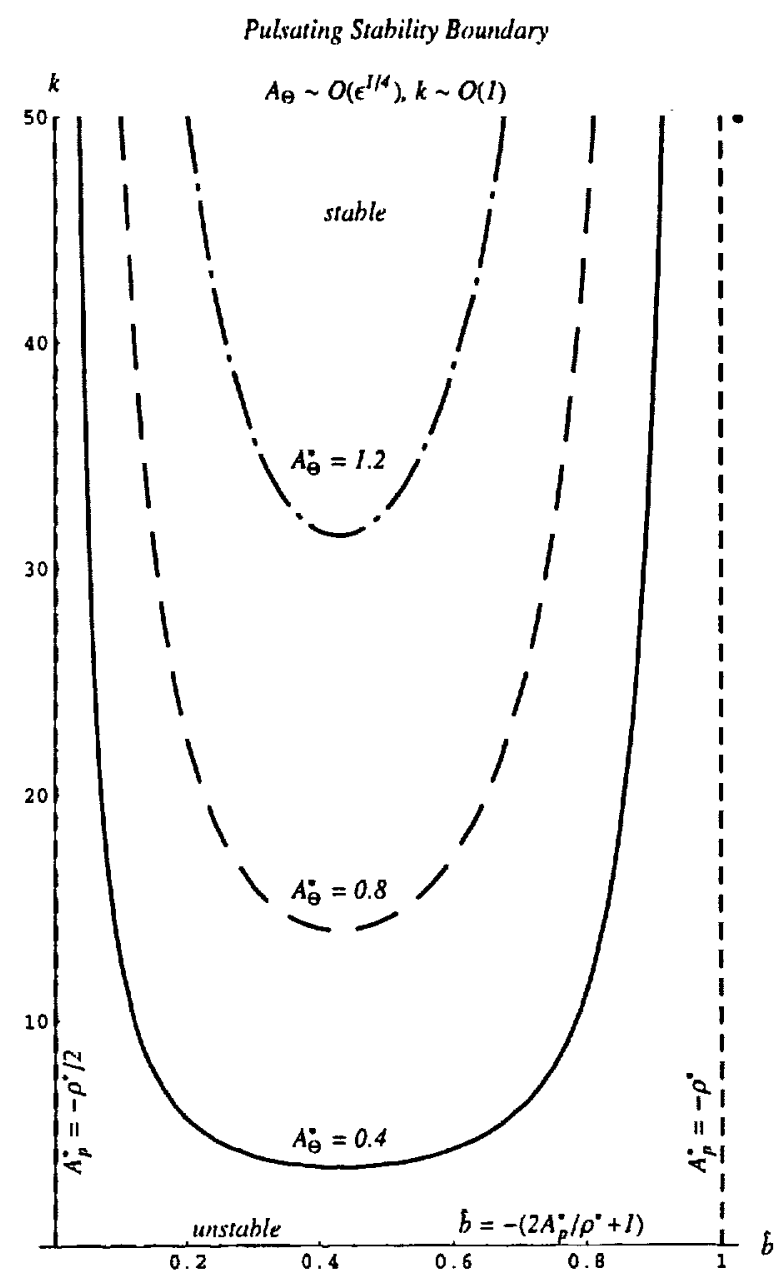

Figure 2. Pulsating hydrodynamic stability boundaries for $k \sim O(1)$ and $A_{\Theta} \sim O\left(\epsilon^{1 / 4}\right)$.

A composite asymptotic solution for the neutral stability boundary in the regime $A_{\Theta} \sim O\left(\epsilon^{1 / 4}\right)$ is thus obtained by matching the cellular and pulsating boundaries in the far outer wavenumber regime, where the former is given by Eq. (61) and the latter is given trivially by $A_{p}^{*}=-\rho^{*}$, with the appropriate solution branch of Eq. (72) in the $\mathrm{O}(1)$ wavenumber region. In particular, we denote the two so-

*These results correct an erroneous conclusion drawn in a previous study, ${ }^{12}$ where an algebraic error in a similar, but less formal, calculation incorrectly suggested that the pulsating boundary recedes (to more negative values of $A_{p}^{*}$ ) as $A_{\Theta}^{*}$ increases, leaving the cellular boundary unaffected. 
lution branches of Eq. (72), which correspond to the portions of Figure 2 that lie to the left and to the right of the minimum at $\hat{b}=\hat{b}_{c}$, by $A_{p}^{*(o, u)}(k)$ and $A_{p}^{*(o, l)}(k)$, where the superscript "o" denotes, as before, the outer, or $O(1)$, wavenumber region and the superscripts " $\mathrm{u}$ " and "l" denote the upper and lower (rotate Figure 2 by $-90^{\circ}$ ) portions of the double-valued pulsating boundary $A_{p}^{*}(k)$. These branches are given implicitly by Eq. (73b), where, by definition, $\hat{b}=-\left(2 A_{p}^{*} / \rho^{*}+1\right)$. Along the upper branch, $A_{p}^{*(o, u)} \rightarrow-\rho^{*} / 2$ (i.e., $\hat{b} \rightarrow 0$ ) as $k \rightarrow \infty$, which can be matched with Eq. (61) since, from Eq. (61), $A_{p}^{*(f)} \rightarrow-\rho^{*} / 2$ as $k_{f} \rightarrow 0$. Similarly, $A_{p}^{*(o, l)} \rightarrow-\rho^{*}$ (i.e., $\hat{b} \rightarrow 1$ ) as $k \rightarrow \infty$, which clearly matches the pulsating boundary $A_{p}^{*}=-\rho^{*}$ in the far outer wavenumber region. As a result, a leadingorder composite stability boundary spanning both the outer and far outer wavenumber regions is given by

$$
A_{p}^{*}(k) \sim \begin{cases}A_{p}^{*(o, u)}(k)+\epsilon \rho^{* 2} \gamma k / 2, & A_{p}^{*} \geq-5 \rho^{*} / 7 \\ A_{p}^{*(o, l)}(k), & A_{p}^{*} \leq-5 \rho^{*} / 7,\end{cases}
$$

Figure 3. Composite hydrodynamic stability boundary for $A_{\ominus} \sim O\left(\epsilon^{1 / 4}\right)$. where the second term in the top expression has been expressed in terms of the outer wavenumber variable $k$. This composite boundary is shown in Figure 3. We note, based on the above construction, that the lower branch of $\mathrm{Eq}$. (74) is a pulsating boundary for all wavenumbers, whereas the upper branch transitions from a pulsating boundary for $O(1)$ wavenumbers to a cellular boundary for $\mathrm{O}\left(\epsilon^{-1}\right)$ wavenumbers. Indeed, from Eq. (70b), we observe that in the outer wavenumber regime, the size of the upper region of oscillatory instability, which is bounded below by the upper branch of the pulsating stability boundary and above by the region of nonoscillatory instability beyond the old cellular boundary $A_{p}^{*} \sim-\rho^{*} / 2$ for $A_{\ominus}^{*}=0$, shrinks to zero as $k$ becomes large.

\section{Evolution of the Pulsating Boundary for $A_{\Theta}^{*} \ll 1$}

The nature of the evolution, as $A_{\Theta}^{*}$ decreases, of the pulsating stability boundary depicted in Figure 2 to that shown in Figure 1 for the case $A_{\Theta}^{*}=0$ may be determined by analyzing the dispersion relation for smaller order-of-magnitude wavenumbers and appropriately rescaled values of $A_{\ominus}$. In particular, the results (70b) and (73b) suggest that if we consider $k \sim \mathrm{O}\left(\epsilon^{a}\right)$, then $A_{\ominus}^{*} \sim \mathrm{O}\left(\epsilon^{a / 2}\right)$ and $i \omega_{0} \sim \mathrm{O}\left(\epsilon^{\mathfrak{a}}\right)$, where the latter imply that $A_{\ominus}$ and $i \omega$

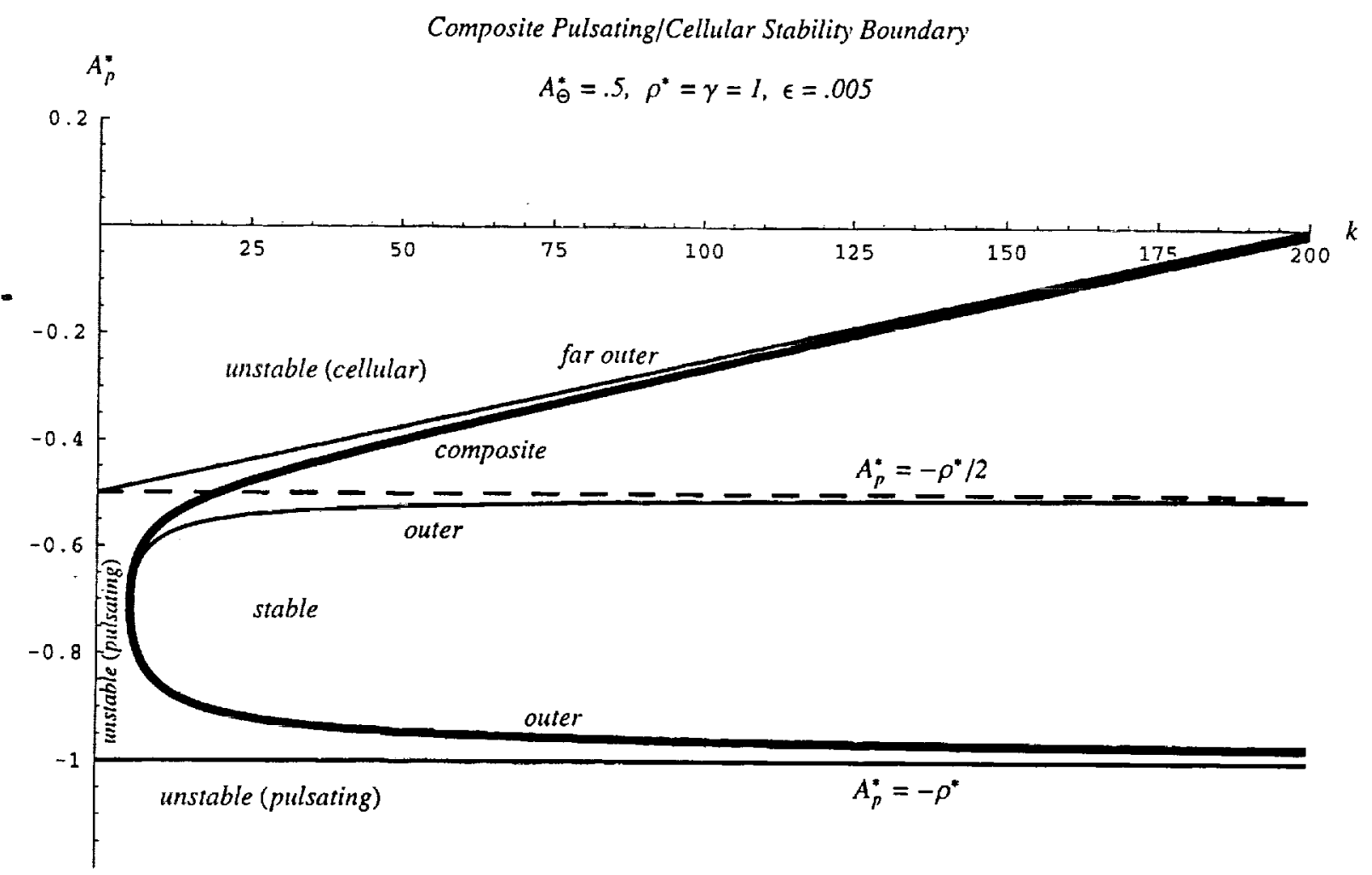


are $O\left(\epsilon^{(2 a+1) / 4}\right)$ and $O\left(\epsilon^{(2 a-1) / 2}\right)$, respectively. A preliminary analysis then suggests that qualitative deviations from the $\mathrm{C}$-shaped nature of the pulsating boundary exhibited in Figure 2 occur for wavenumbers of order $\epsilon^{1 / 2}$, which is another intermediate wavenumber scale that lies between the inner and outer wavenumber regions that were defined in.the previous section. The above analysis is thus repeated for $k=\hat{k} \epsilon^{1 / 2}$ and $A_{\Theta}=\hat{A}_{\Theta} \epsilon^{1 / 2}$, the other parameters remaining unchanged from their previously assumed orders of magnitude. Consequently, in place of Eq. (65), a solution for the dispersion relation is now sought in the form

$$
i \omega \sim i \hat{\omega}_{0}+\epsilon^{1 / 4} i \hat{\omega}_{1}+\epsilon^{1 / 2} i \hat{\omega}_{2}+\cdots .
$$

Substituting Eq. (75) and the new scalings introduced above into Eqs. (37), (40) and (53), the new expanded forms of $p, s$ and $b_{8}$ are given by

$$
\begin{gathered}
p \sim \hat{p}_{0}+\hat{p}_{1} \epsilon^{1 / 4}+\hat{p}_{2} \epsilon^{1 / 2}+\cdots, \quad s \sim \mathrm{O}(\epsilon), \quad \\
b_{8} \sim-\frac{\left(i \hat{\omega}_{0}\right)^{2} \rho^{*}+\hat{k}^{2}}{2 \rho^{*} \hat{k}} \epsilon^{-1 / 2}-\frac{1}{\hat{k}}\left(i \hat{\omega}_{0}\right)\left(i \hat{\omega}_{1}\right) \epsilon^{-1 / 4} \\
-\frac{1}{2 \hat{k}}\left[\left(i \hat{\omega}_{1}^{2}\right)+2\left(i \hat{\omega}_{0}\right)\left(i \hat{\omega}_{2}\right)\right] \epsilon^{0}+\cdots,
\end{gathered}
$$

where

$$
\begin{gathered}
\hat{p}_{0}=\frac{1}{2}\left[1+\left(i \hat{\omega}_{0}\right)^{1 / 2}\right], \quad \hat{p}_{1}=\frac{1}{4} i \hat{\omega}_{1}\left(1+4 i \hat{\omega}_{0}\right)^{-1 / 2}, \\
\hat{p}_{2}=\frac{1}{4} i \hat{\omega}_{2}\left(1+4 i \hat{\omega}_{0}\right)^{-1 / 2} .
\end{gathered}
$$

As a result, Eqs. (57) and (67) give the corresponding expansion of $b_{10}$ as

$$
\begin{gathered}
\epsilon_{10} \sim \frac{A_{p}^{*}}{\hat{A}_{\Theta}}\left\{\frac{\hat{k}}{\rho^{*}}+\frac{i \hat{\omega}_{0}}{2 \hat{k}^{2}}\left[\left(i \hat{\omega}_{0}\right)^{2} \rho^{*}-3 \hat{k}^{2}\right] \epsilon^{1 / 2}+\cdots\right\} \\
+\frac{\epsilon^{0}}{\hat{A}_{\ominus}}\left\{-\frac{1}{2 \hat{k}}\left[\left(i \hat{\omega}_{0}\right)^{2} \rho^{*}-\hat{k}^{2}\right]-\frac{\rho^{*}}{\hat{k}}\left(i \hat{\omega}_{0}\right)\left(i \hat{\omega}_{1}\right) \epsilon^{1 / 4}\right. \\
+\frac{\rho^{*}}{2 \hat{k}}\left[\frac{i \hat{\omega}_{0}}{\hat{k}}\left\{\left(i \hat{\omega}_{0}\right)^{2} \rho^{*}-3 \hat{k}^{2}\right\}-\left(i \hat{\omega}_{1}\right)^{2}\right. \\
\left.\left.-2\left(i \hat{\omega}_{0}\right)\left(i \hat{\omega}_{2}\right)\right] \epsilon^{1 / 2}+\cdots\right\} \\
\sim \hat{b}_{10}^{(0)}+\hat{b}_{10}^{(1)} \epsilon^{1 / 4}+\hat{b}_{10}^{(2)} \epsilon^{1 / 2}+\cdots .
\end{gathered}
$$

Substituting these expansions into the dispersion relation (59), one obtains from the leading $O\left(\epsilon^{0}\right)$ terms in that equation the result $\hat{b}_{10}^{(0)}=0$, or, according to Eq. (79),

$$
\left(i \hat{\omega}_{0}\right)^{2}=\left(\frac{\hat{k}}{\rho^{*}}\right)^{2}\left(2 A_{p}^{*}+\rho^{*}\right) .
$$

Comparing Eq. (80) with Eq. (70b), it is thus seen that the same leading-order result is obtained for $i \hat{\omega}_{0}(\hat{k})$ for $A_{\Theta} \sim \mathrm{O}\left(\epsilon^{1 / 2}\right)$ as was obtained for $i \omega_{0}(k)$ for $A_{\Theta} \sim \mathrm{O}\left(\epsilon^{1 / 4}\right)$. In particular, $i \hat{\omega}_{0}$ is real and positive for $A_{p}^{*}>-\rho^{*} / 2$, and is pure imaginary for $A_{p}^{*} \leq-\rho^{*} / 2$. Thus, the prediction of cellular instability is recovered in the former case, but stability in the latter region can only be ascertained by calculating the next nontrivial term in the expansion (75).

Collecting terms of $\mathrm{O}\left(\epsilon^{1 / 4}\right)$ in the expanded version of Eq. (59), it is concluded that $\hat{b}_{10}^{(1)}=0$, which, from Eq. (79), implies that $i \hat{\omega}_{1}=0$. Proceeding to the next order, we collect terms of $O\left(\epsilon^{1 / 2}\right)$ in $\mathrm{Eq}$. (59), which gives rise to the nontrivial equation

$$
\hat{b}_{10}^{(2)}\left(1-c-\hat{p}_{0}\right)+\left(2 \hat{k}+\rho^{*} \hat{b}_{8}^{(0)}\right)\left(1+\frac{1-\hat{p}_{0}}{i \hat{\omega}_{0}}\right)=0,
$$

where, from Eq. $(77), \hat{b}_{8}^{(0)}=-\left(i \hat{\omega}_{0}\right)\left(i \hat{\omega}_{2}\right) / \hat{k}$. Upon substituting the expressions for $\hat{b}_{10}^{(2)}$ and $\left(i \hat{\omega}_{0}\right)^{2}$ from Eqs. (79) and (80), we obtain the expression for $i \hat{\omega}_{2}$ as

$$
\begin{aligned}
i \hat{\omega}_{2}= & \hat{k}\left(A_{p}^{*}-\rho^{*}\right)\left[A_{p}^{*}+\rho^{*}\right. \\
& \left.-\frac{\hat{A}_{\ominus} \rho^{* 2}}{\hat{k}\left(2 A_{p}^{*}+\rho^{*}\right)}\left(\frac{1+2 i \hat{\omega}_{0}-\sqrt{1+4 i \hat{\omega}_{0}}}{1-2 c-\sqrt{1+4 i \hat{\omega}_{0}}}\right)\right],
\end{aligned}
$$

where, from Eq. (80), $i \hat{\omega}_{0}=\left(\hat{k} / \rho^{*}\right)\left(2 A_{p}^{*}+\rho^{*}\right)^{1 / 2}=$ $\left(\hat{k} / \rho^{*}\right)\left[-\left(2 A_{p}^{*}+\rho^{*}\right)\right]^{1 / 2} e^{i \pi / 2}$, the second equality denoting the principal root when $A_{p}^{*}<-\rho^{*} / 2$. The expressions (81b) and (71b) for the two growth-rate corrections $i \hat{\omega}_{2}$ and $i \omega_{2}$ in their respective wavenum-

- ber regimes collapse to the same result in the limit of zero thermal sensitivity, yielding the same pulsating stability boundary $A_{p}^{*}=-\rho^{*}$ as before. However, for nonzero $\hat{A}_{\Theta}$, the forms of these two results differ, leading to qualitative differences in the corresponding neutral stability boundaries. In particular, it will be shown below that the pulsating neutral stability boundary obtained from Eq. (81b) approaches that shown in Figure 1 (i.e., $A_{p}^{*}=-\rho^{*}$ ) in the limit of zero thermal sensitivity, whereas it approaches the form shown in Figure 2 as $\hat{A}_{\Theta}$ becomes large. That is, the evolution between these two limiting forms occurs on the $\mathrm{O}\left(\epsilon^{1 / 2}\right)$ wavenumber scale for $\mathrm{O}\left(\epsilon^{1 / 2}\right)$ values of the thermal sensitivity parameter $A_{\Theta}$.

Setting the real part of $i \bar{\omega}_{2}$ to zero, the equation governing the (pulsating) neutral stability boundary $A_{p}^{*}(\hat{k})$ in the present wavenumber regime is deter- 
mined from Eq. (81b) as

$$
\begin{aligned}
& A_{p}^{*}+\rho^{*} \\
& -\frac{\hat{A}_{\Theta} \rho^{* 2}}{\hat{k}\left(2 A_{p}^{*}+\rho^{*}\right)} \mathcal{R} e\left\{\frac{1+2 i \hat{\omega}_{0}-\sqrt{1+4 i \hat{\omega}_{0}}}{1-2 c-\sqrt{1+4 i \hat{\omega}_{0}}}\right\}=0,
\end{aligned}
$$

which, since $i \hat{\omega}_{0}$ depends on $A_{p}^{*}$ and $\hat{k}$, is a highly nonlinear equation for $A_{p}^{*}(\hat{k})$. Thus, in order to determine the nature of the stability boundary as a function of $\hat{A}_{\Theta}$, it is useful to consider certain limiting cases. We first consider possible solutions of Eq. (82) in the large (scaled) wavenumber regime $\hat{k} \gg 1$. In that limit, we observe from Eq. (70b) that $i \hat{\omega}_{0} \sim O(\hat{k}) \gg 1$, and thus a first approximation to Eq. (81) in this regime is given by

$$
A_{p}^{*}+\rho^{*}-\frac{\hat{A}_{\Theta} \rho^{* 2}}{\hat{k}\left(2 A_{p}^{*}+\rho^{*}\right)} \mathcal{R} e\left\{-\sqrt{\hat{i} \hat{\omega}_{0}}\right\} \sim 0,
$$

or, using the expression for the principal root $i \hat{\omega}_{0}$ given below $\mathrm{Eq}$. (81b),

$A_{p}^{*}+\rho^{*}-\frac{1}{2} \sqrt{2} \rho^{* 3 / 2} \hat{k}^{-1 / 2} \hat{A}_{\ominus}\left[-\left(2 A_{p}^{*}+\rho^{*}\right)\right]^{-3 / 4} \sim 0$,

which, since $\hat{k}^{-1 / 2} \hat{A}_{\Theta}=\left(k / \epsilon^{1 / 2}\right)^{-1 / 2}\left(A_{\Theta} / \epsilon^{1 / 2}\right)=$ $k^{-1 / 2}\left(A_{\ominus} / \epsilon^{1 / 4}\right)=k^{-1 / 2} A_{\Theta}^{*}$, is identical to Eq. (72). According to the discussion of Eq. (72) above, the turning point in the corresponding neutral stability boundary exhibited in Figure 2 occurs at the value $k_{c} \propto A_{\Theta}^{* 2}$. Thus, we conclude that Eq. (83b) is a valid approximation for Eq. (82) in the large (scaled) wavenumber regime $\hat{k} \gg 1$ provided $\hat{A}_{\Theta}^{2} \gg 1$, where the latter is a necessary condition to insure that $\hat{k}$ is large everywhere along the stability boundary described by Eq. (83b). For such values of $\hat{A}_{\Theta}$, the pulsating stability boundary for large $\hat{k}$ approaches that illustrated in Figure 2 (with $k$ replaced by $\hat{k}$ and $A_{\Theta}^{*}$ replaced with $\left.\hat{A}_{\Theta}\right)$.

In addition to the boundary described by Eq. (83b) and Figure 2 for large $\hat{k}$ and $\hat{A}_{\ominus}$, there is another pulsating branch that is present for small wavenumbers, as suggested by the observation that there exists a solution of Eq. (82) such that $A_{p}^{*} \rightarrow$ $-\rho^{*}$ as $\hat{k} \rightarrow 0$, irrespective of the magnitude of $\hat{A}_{\ominus}$. To simplify the analysis and further discussion, we mainly consider a simplified form of Eq. (82) by restricting further consideration to the reasonable limit in which the heat-capacity ratio $c$ is small. Since $1+2 i \hat{\omega}_{0}-\sqrt{1+4 i \hat{\omega}_{0}}=\left(1-\sqrt{1+4 i \hat{\omega}_{0}}\right)^{2} / 2$, a first approximation to Eq. (82) in that limit is given by

$$
\begin{gathered}
A_{p}^{*}+\rho^{*}+\frac{\hat{A}_{\ominus \rho^{* 2}}}{2 \hat{k}\left(2 A_{p}^{*}+\rho^{*}\right)} \mathcal{R} e\left\{\sqrt{1+4 i \hat{\omega}_{0}}-1\right\} \sim 0, \\
c \rightarrow 0
\end{gathered}
$$

which clearly gives the same limiting approximation (83a) for large $\hat{k}$. To analyze Eq. (84) for arbitrary $\hat{k}$, it is convenient to convert it to an alternative form as follows. Writing $I+4 i \omega_{0}$ in the polar form $1+4 i \hat{\omega}_{0}=r \cos \vartheta+i \sin \vartheta$, where $r^{2}=1+16 \hat{\omega}_{0}^{2}$, $\cos \vartheta=1 / r$ and $\sin \vartheta=4 \hat{\omega}_{0} / r$, Eq. (84) may be rewritten as

$$
2 \hat{\omega}_{0}^{2}\left(A_{p}^{*}+\rho^{*}\right)=\hat{k} \hat{A}_{\Theta}\left[\sqrt{\frac{\left(1+16 \hat{\omega}_{0}^{2}\right)^{1 / 2}+1}{2}}-1\right],
$$

where, from Eq. $(80), \hat{\omega}_{0}^{2}=-\left(\hat{k} / \rho^{*}\right)^{2}\left(2 A_{p}^{*}+\rho^{*}\right)$. Substituting this expression for $\hat{\omega}_{0}^{2}$ into Eq. (85) and rearranging/squaring the latter twice so as to remove the fractional powers, a polynomial equation for the inverse relation $\hat{k}\left(A_{p}^{*} ; \hat{A}_{\ominus}\right)$ is obtained as

$$
\begin{aligned}
& 4 \hat{k}^{3}\left(2 A_{p}^{*}+\rho^{*}\right)^{3}\left(A_{p}^{*}+\rho^{*}\right)^{4} \\
&-8 \hat{k}^{2} \rho^{* 2} \hat{A}_{\Theta}\left(2 A_{p}^{*}+\rho^{*}\right)^{2}\left(A_{p}^{*}+\rho^{*}\right)^{3} \\
&+\hat{k}\left[5 \rho^{* 4} \hat{A}_{\Theta}^{2}\left(2 A_{p}^{*}+\rho^{*}\right)\left(A_{p}^{*}+\rho^{*}\right)^{2}+\rho^{* 6} \hat{A}_{\Theta}^{4}\right] \\
& \quad-\rho^{* 6} \hat{A}_{\Theta}^{3}\left(A_{p}^{*}+\rho^{*}\right)=0,
\end{aligned}
$$

or, introducing $\hat{b}=-\left(2 A_{p}^{*} / \rho^{*}+1\right)$ as before,

$$
\begin{aligned}
& \hat{k}^{3} \hat{b}^{3}(1-\hat{b})^{4}+4 \hat{k}^{2} \hat{A}_{\Theta} \hat{b}^{2}(1-\hat{b})^{3} \\
& \quad+\bar{k}\left[5 \hat{A}_{\Theta}^{2} \hat{b}(1-\hat{b})^{2}-4 \hat{A}_{\Theta}^{4} / \rho^{*}\right]+2 \hat{A}_{\Theta}^{3}(1-\hat{b})=0 .
\end{aligned}
$$

An even more compact version is obtained by defining $\tilde{k}=\hat{b}(1-\hat{b}) \hat{k} / \hat{A}_{\Theta}$, giving the cubic equation

$$
\vec{k}^{3}+4 \bar{k}^{2}+(5-\alpha) \bar{k}+2=0,
$$

where $\alpha(\hat{b})=4 \hat{A}_{\Theta}^{2} /\left[\rho^{*} \hat{b}(1-\hat{b})^{2}\right]$. However, since it is the relation $A_{p}^{*}\left(\hat{k} ; \hat{A}_{\Theta}\right)$, or equivalently, $\hat{k}\left(\hat{b} ; \hat{A}_{\Theta}\right)$, that is ultimately desired, we shall mainly consider the form given by Eq. (86b).

In the regime $\hat{A}_{\ominus} \gg 1$ there exists a solution $\hat{k} \sim O\left(\hat{A}_{\Theta}^{2}\right)$ as suggested above. More precisely, this solution of Eq. (86b) may be sought in the form

$$
\hat{k} \sim \hat{A}_{\Theta}^{2}\left(\hat{k}_{0}+\hat{A}_{\Theta}^{-1} \hat{k}_{1}+\hat{A}_{\Theta}^{-2} \hat{k}_{2}+\cdots\right) .
$$

whence, upon substitution of this expansion into Eq. (86b), the coefficients $\hat{k}_{i}$ are determined recursively as

$\hat{k}_{0}=2 \hat{b}^{-3 / 2}(1-\hat{b})^{-2} / \rho^{* 1 / 2}, \quad \hat{k}_{1}=-2 \hat{b}^{-1}(1-\hat{b})^{-1}$, 
and so forth. As expected, the leading-order approximation $\hat{k} \sim \hat{A}_{\theta}^{2} \hat{k}_{0}$ is identical in form to the result (73b) and the next term in the above expansion thus provides a negative correction to this result. On the other hand, for large $\hat{A}_{\ominus}$ there also exists a solution $\hat{k} \ll 1$ of the form

$$
\hat{k} \sim \hat{A}_{\Theta}^{-1}\left(\bar{k}_{0}+\hat{A}_{\Theta}^{-1} \bar{k}_{1}+\hat{A}_{\Theta}^{-2} \bar{k}_{2}+\cdots\right) .
$$

Substituting the latter into Eq. (86b), the $\bar{k}_{i}$ are also determined recursively, the first few of which are given as

$\bar{k}_{0}=\rho^{*}(1-\hat{b}) / 2, \quad \bar{k}_{1}=0, \quad \bar{k}_{2}=5 \rho^{* 2} \hat{b}(1-\hat{b})^{3} / 8$

where the last of these provides, for $0<\hat{b}<1$, a strictly positive correction to the leading-order approximation $\hat{k} \sim\left(2 \hat{A}_{\Theta}\right)^{-1} \rho^{*}(1-\hat{b})$. Although the branches described by Eqs. (87) and (89) were obtained for $\hat{A}_{\Theta} \gg 1$, they remain reasonably accurate representations of the actual branches even for $O(1)$ values of this parameter, as shown in Figures $4 a, b$ (discussed immediately below).

As $\hat{A}_{\Theta}$ decreases through $O(1)$ values, the turning point in the stability boundary for large $\hat{k}$ occurs for smaller values of $\hat{k}$, and eventually the expansion for large $\hat{k}$ ceases to be a valid approximation of Eq. (86b). Simultaneously, the branch corresponding to small values of $\hat{k}$ reaches larger values of $\hat{k}$ such that the above expansion for small $\hat{k}$ ceases to be valid as well. These two branches eventually intersect, restoring a region of stability for a range of negative values of $A_{p}^{*}$. This development is illustrated in Figures $4 \mathrm{a}-\mathrm{f}$, which were obtained directly from the exact roots of the cubic equation (86b) with respect to $\hat{k}$ [for comparison, we also indicate the asymptotic representation for the upper branch corresponding to one- and two-term truncations of Eq. (87)]. We observe that after the two pulsating branches intersect (as, for example, in Figures 4c-e), there exist two critical values of $\hat{k}$ corresponding to the onset of pulsating instability at corresponding critical values of $\hat{b}$, or equivalently, $A_{p}^{*}$. That is, in this range of temperature sensitivities, a pulsating hydrodynamic instability occurs for either sufficiently small or sufficiently large negative values of $A_{p}^{*}$ as either the left or right (upper or lower in the rotated frame of reference) stability boundary is crossed. As $\hat{A}_{\ominus}$ decreases further, the separated branches move further apart until ultimately, in the limit $\hat{A}_{\ominus} \rightarrow 0$, we recover from Eqs. (86) the two roots $\hat{b}=0$ and $\hat{b}=1$ corresponding to the cellular $\left(A_{p}^{*}=-\rho^{*} / 2\right)$ and pulsating $\left(A_{p}^{*}=-\rho^{*}\right)$ stability boundaries in the present $\left[k \sim \mathrm{O}\left(\epsilon^{1 / 2}\right)\right]$ wavenumber regime. Thus, as the temperature-sensitivity parameter $A_{\Theta}$ decreases to small values on the scale $A_{\ominus} / A_{p} \sim \mathrm{O}\left(\epsilon^{-1 / 2}\right) \sim 50$, the region of hydrodynamic stability depicted in Figure 1 is recovered.

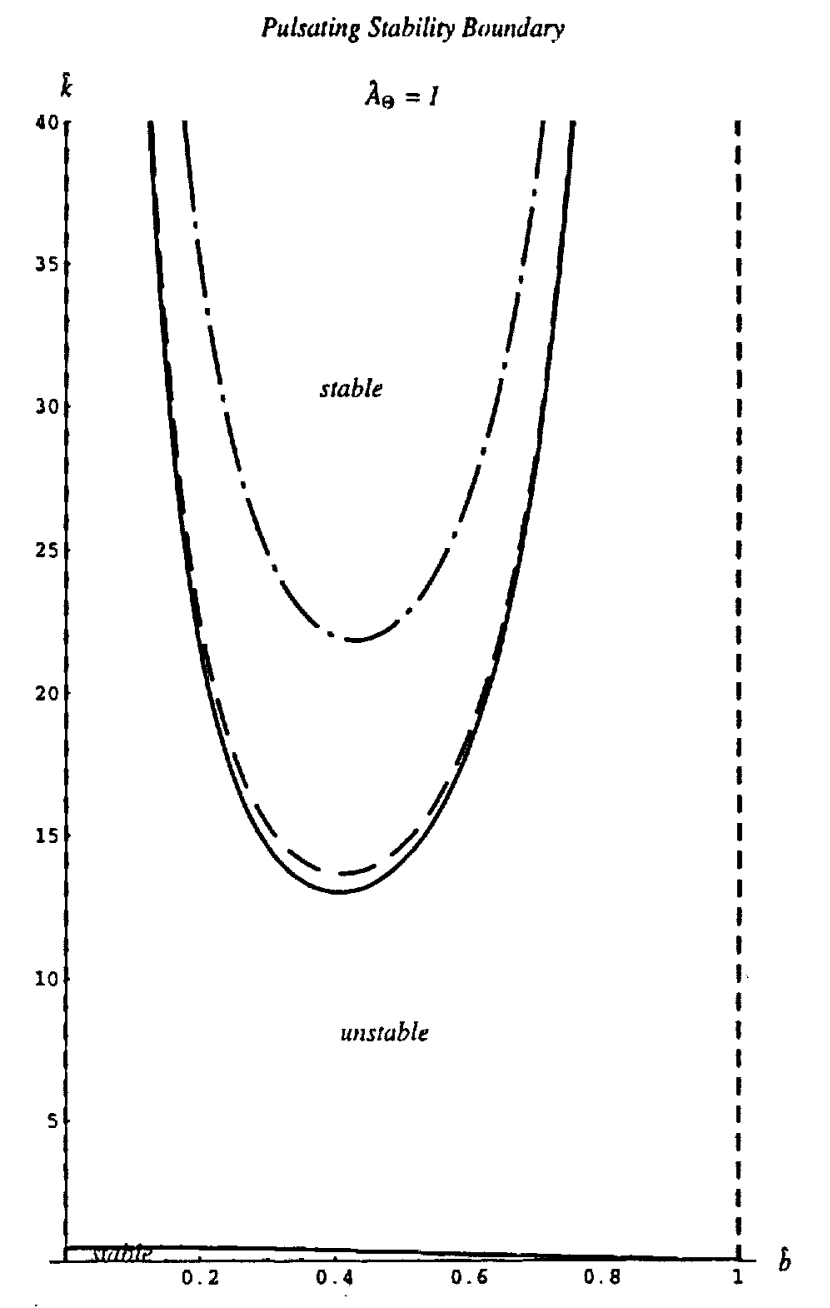

Figure 4a

Figures 4a-f. Pulsating hydrodynamic stability boundaries for $k=\hat{k} \epsilon^{1 / 2} \sim O\left(\epsilon^{1 / 2}\right)$ and decreasing values of $A_{\Theta}=\hat{A}_{\Theta} \epsilon^{1 / 2} \sim O\left(\epsilon^{1 / 2}\right)$. The broken curves denote one- and two-term asymptotic expansions of the upper boundary for $\hat{A}_{\Theta} \gg 1, \hat{k} \gg 1$. (a) $\hat{A}_{\Theta}=1$; (b) $\hat{A}_{\Theta}=2 / 3$; (c) $\hat{A}_{\Theta}=3 / 5$; (d) $\hat{A}_{\Theta}=1 / 2 ;(e) \hat{A}_{\Theta}=1 / 4 ;(f) \hat{A}_{\Theta}=1 / 16$. 


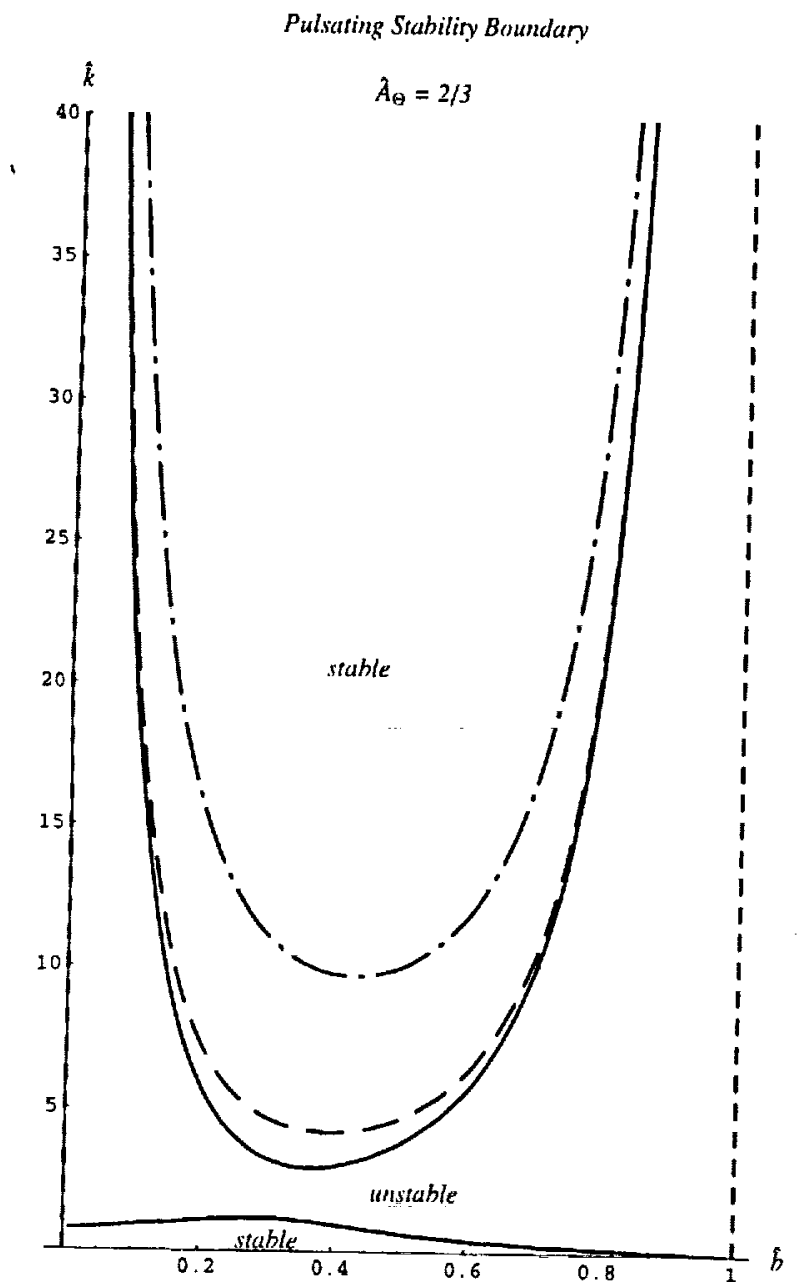

Figure $4 b$

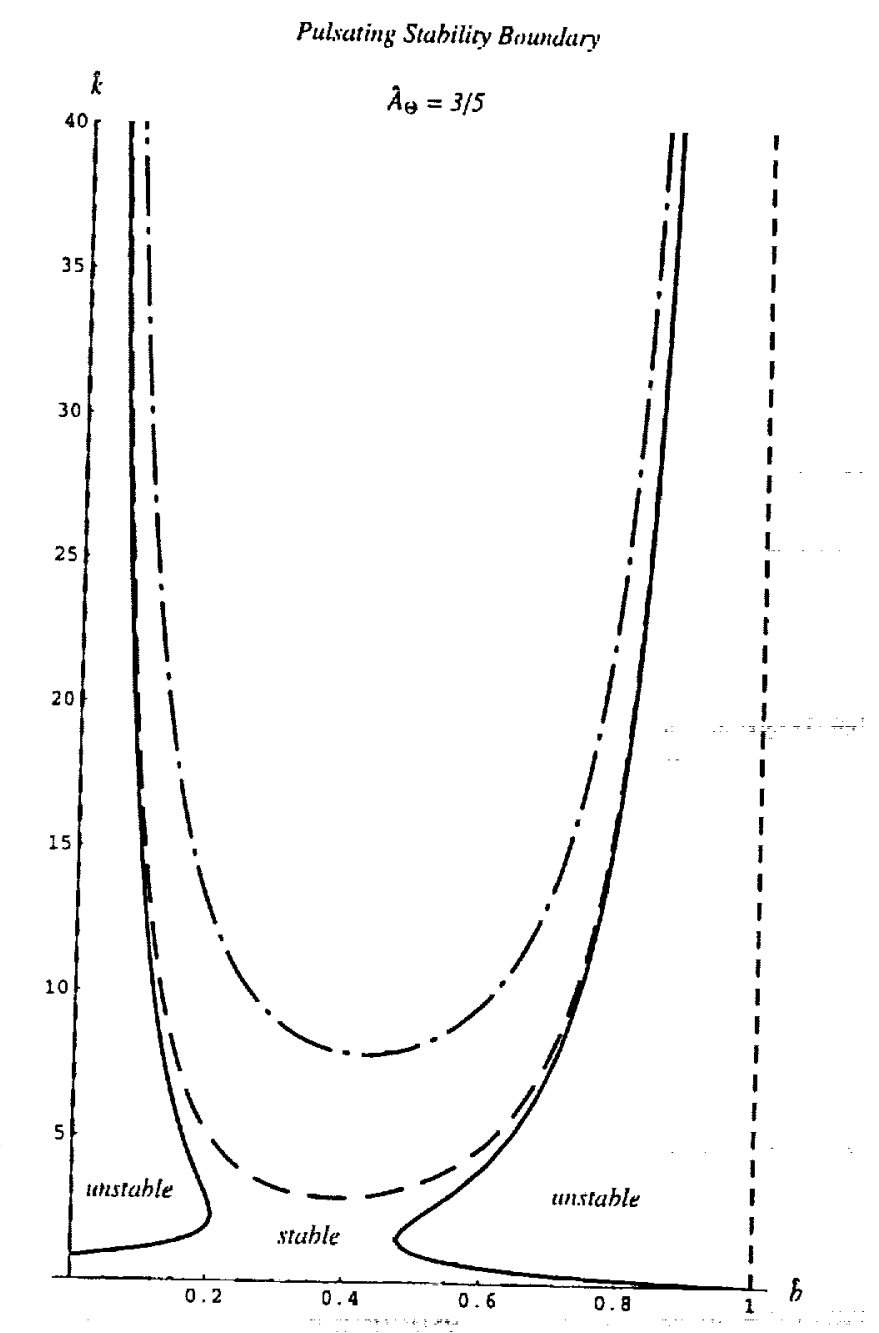

Figure $4 c$ 


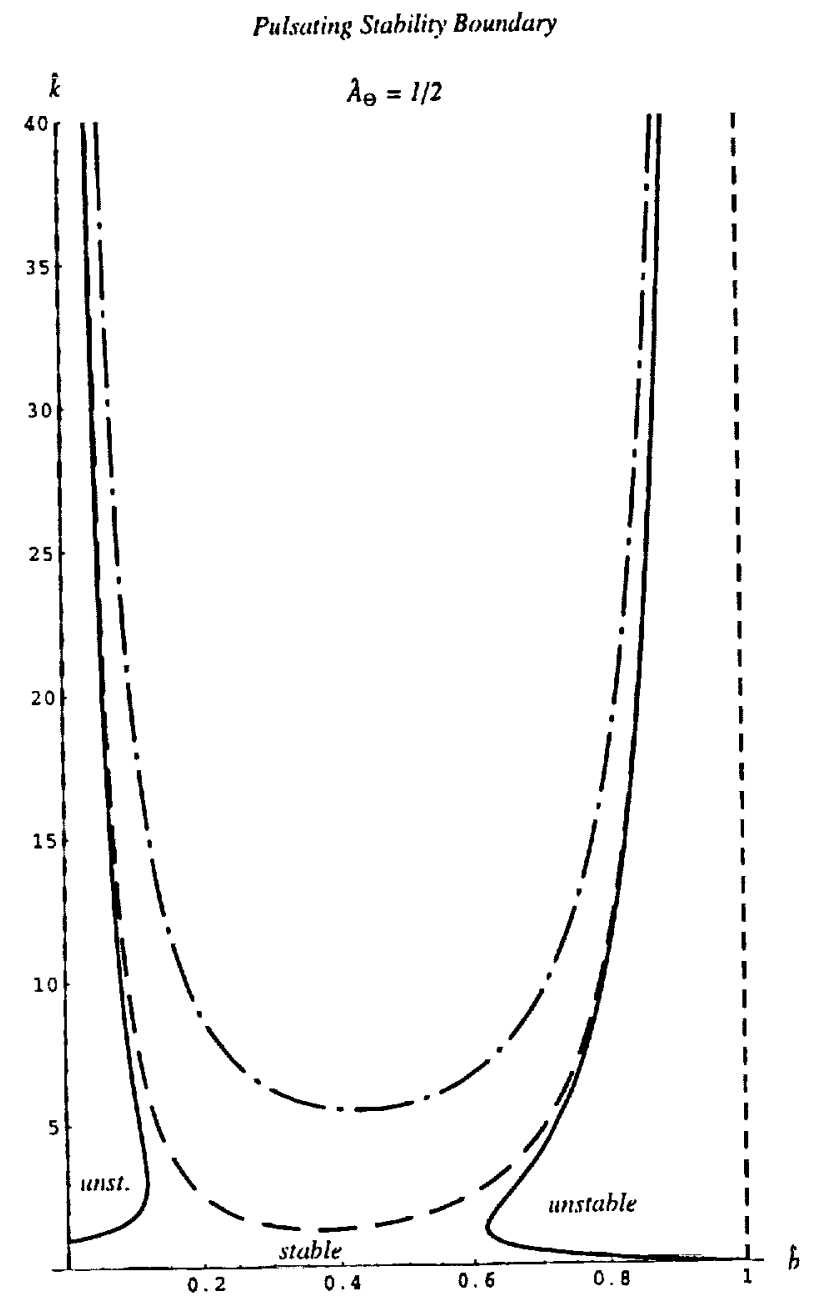

Figure 4d

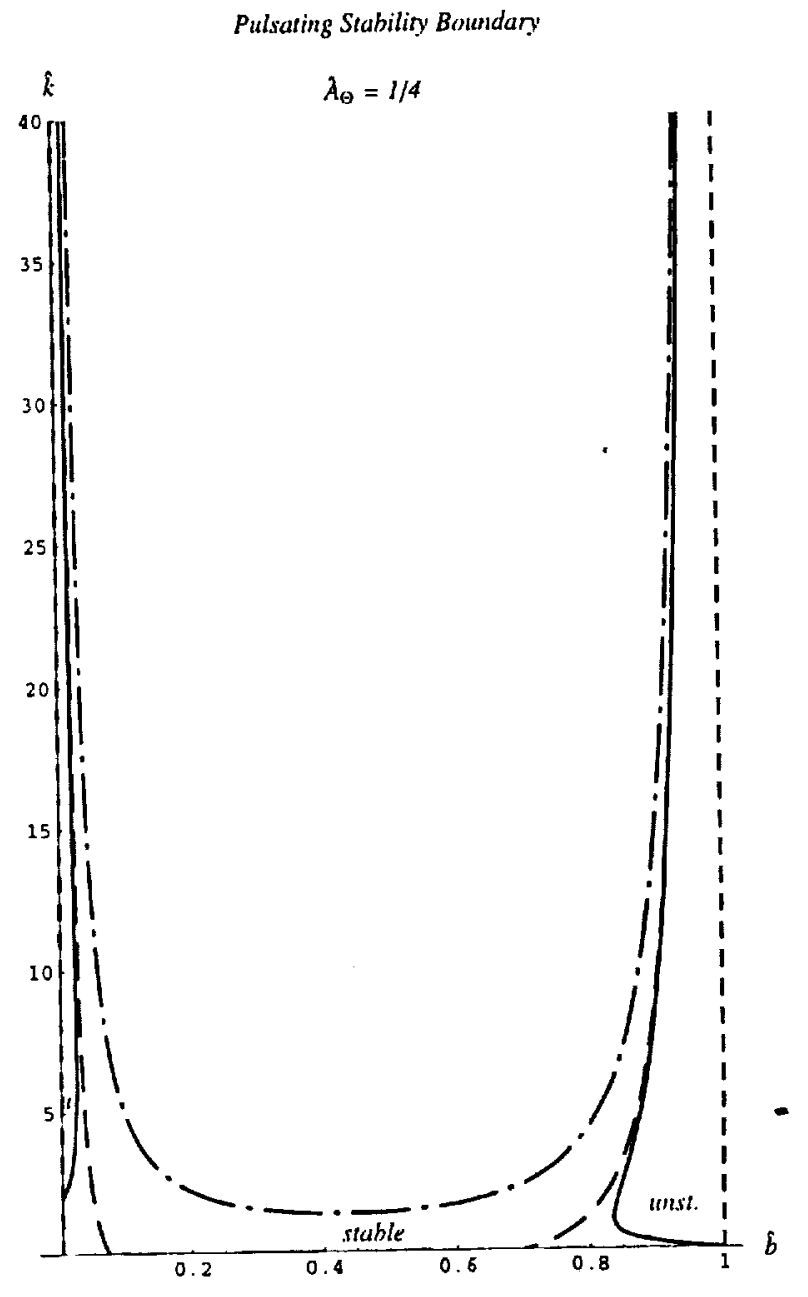

Figure 4e 
Pulscting Stability Bonndary

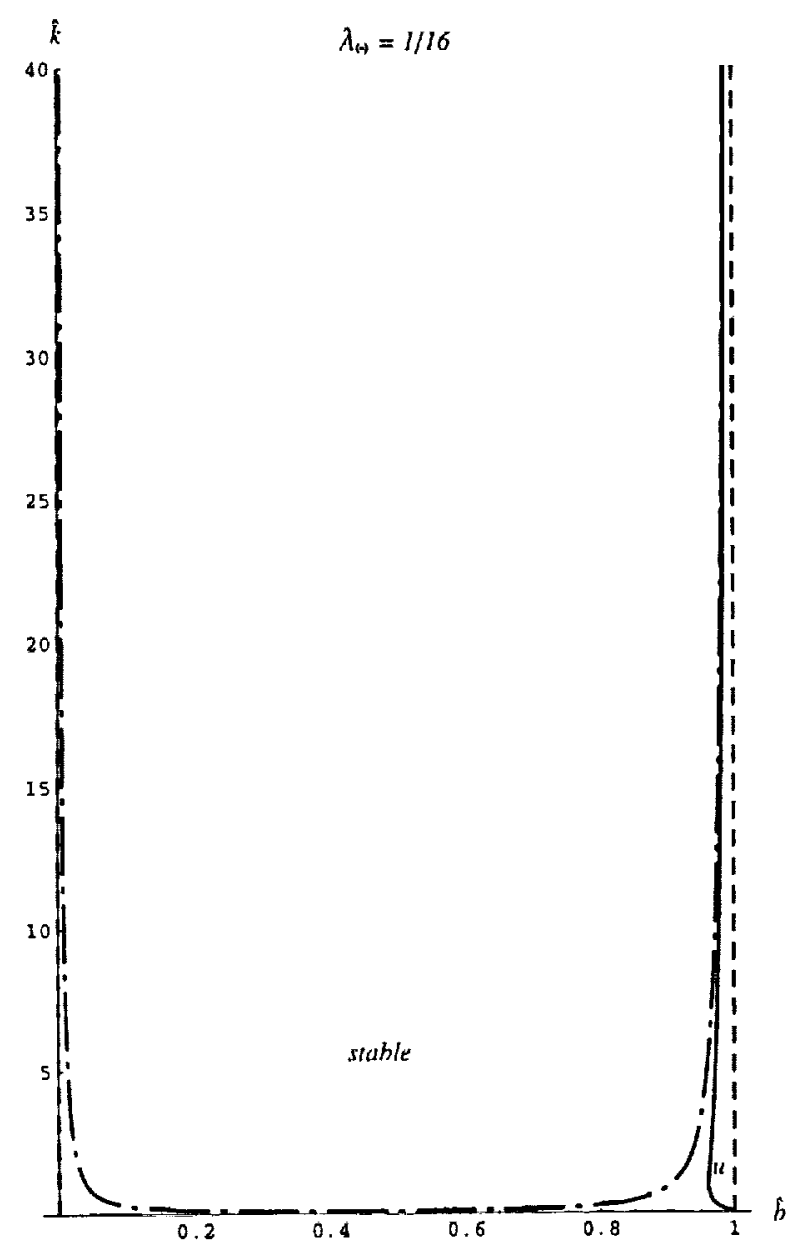

Figure 4f

\section{Viscous Effects}

The above analysis for nonzero $A_{\Theta} \sim \mathrm{O}\left(\epsilon^{1 / 4}\right)$ may be extended to include the effects of viscosity. ${ }^{19}$ Adopting the reasonable scalings $P r_{l}=P \sim O(1)$ and $\mu=\mu^{*} \epsilon \sim O(1)$ for the liquid-phase Prandtl number and the gas-to-liquid viscosity ratio, it was previously shown that modifications to the hydrodynamic cellular stability boundary occur, to a first approximation, only in the far outer wavenumber regime $k \sim O\left(\epsilon^{-1}\right)$, while the leading-order modifications to the pulsating boundary first occur for $O(1)$ wavenumbers. ${ }^{13,14}$ Although these results were obtained by neglecting the thermal coupling analyzed above for the inviscid problem, the same qualitative order-of-magnitude effects remain valid for the present case in which $A_{\Theta}>0$. Similarly, just as it was determined above for the inviscid problem that the effects of thermal coupling are felt in the $O(1)$ wavenumber regime for $A_{\Theta}=A_{\Theta}^{*} \epsilon^{1 / 4} \sim \mathrm{O}\left(\epsilon^{1 / 4}\right)$, a similar result is obtained in the viscous case. Thus, adopting these scalings, we again seek the neutral stability boundary in the $O(1)$ wavenumber regime, anticipating that the primary differences from the inviscid analysis presented above will occur on the right-hand portion of the stability boundaries exhibited in Figure 2. In particular, the dispersion relation $i \omega(k)$ is sought in the form $(65)$; i.e., as $i \omega_{0} \sim \epsilon^{-1 / 2}\left(i \omega_{0}+i \omega_{1} \epsilon^{1 / 4}+i \omega_{2} \epsilon^{1 / 2}+\cdots\right)$. However the added algebraic complexity caused by the inclusion of the various viscous terms leads us to introduce corresponding expansions for the coefficients $b_{i}$ appearing in Eqs. (41) - (51) directly..$^{13,14}$

The details of the corresponding analysis are described elsewhere, ${ }^{19}$ but the main result that is obtained is that the expression for $i \omega_{0}$ is unchanged from the inviscid result (70b), and that $i \omega_{1}$ is again zero. In place of Eq. (71b) for $i \omega_{2}$, however, we obtain the modified expression

$$
\begin{aligned}
i \omega_{2}= & -2 P k^{2}+\frac{k}{\rho^{* 2}}\left(A_{p}^{*}-\rho^{*}\right) \\
& \cdot\left[A_{p}^{*}+\rho^{*}+\rho^{* 3 / 2} k^{-1 / 2} A_{\ominus}^{*}\left(2 A_{p}^{*}+\rho^{*}\right)^{-3 / 4}\right],
\end{aligned}
$$

leading to an implicit equation for the viscous pulsating stability boundary $A_{p}^{*}(k)$ given by

$$
k[(3+\hat{b})(1-\hat{b})+8 P k]^{2}=\frac{2 A_{\Theta}^{*} / \sqrt{\rho^{*}}}{\hat{b}^{3 / 2}}(3+\hat{b})^{2},
$$

where $\hat{b}=-\left(2 A_{p}^{*} / \rho^{*}+1\right)$ as before. In the limit $P \rightarrow 0$, Eq. (92) collapses to the result (73b).

Equation (73b) may be transformed to a cubic equation for $k(\hat{b})$, the single real root of which determines the stability boundary. Typical results are shown in Figure 5, where, in comparison with the corresponding inviscid plot shown in Figure 2, it is seen that the main qualitative difference lies in the asymptote of the right portion of the boundary. In particular, that asymptote is no longer given simply by $\hat{b}=1$, or equivalently, by $A_{p}^{*}=-\rho^{*}$, but by $A_{p}^{*}=-\rho^{*}(1+2 P k)^{1 / 2}$, which is the viscous pulsating boundary in the limit of zero temperature sensitivity. ${ }^{14}$

The pulsating stability boundary (92) may be matched to the far outcr viscous cellular boundary as shown above for the inviscid case, leading to a composite expansion spanning the outer and far outer wavenumber regions. This is exhibited in Figure 6, from which we conclude that viscous effects serve to widen the stable region for wavenumbers bounded away from zero, but do not eliminate the intrinsic pulsating instability that exists for sufficiently small 


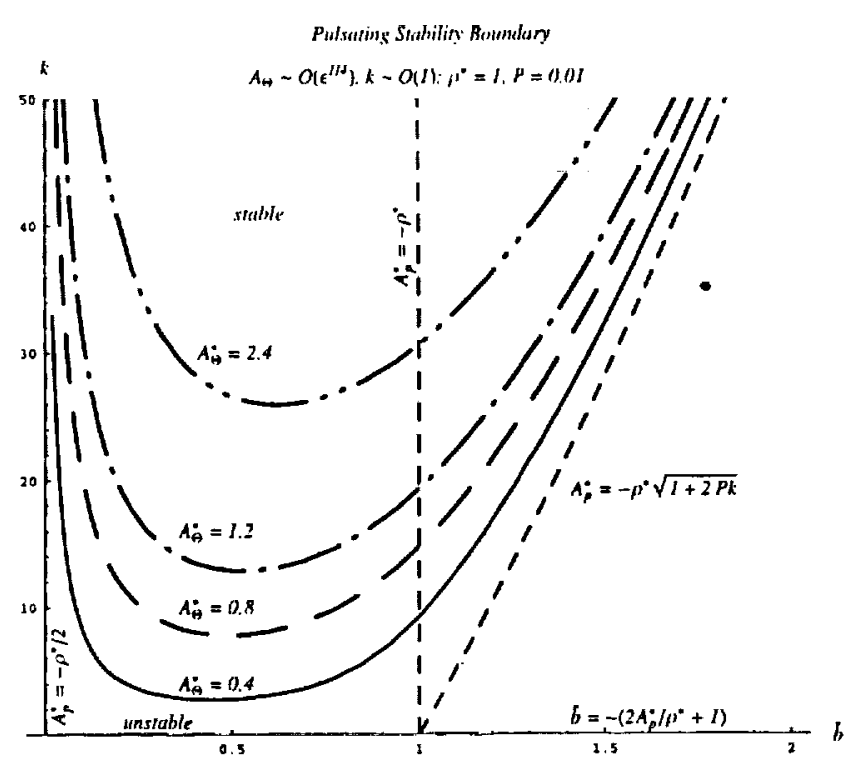

Figure 5. Pulsating hydrodynamic stability boundaries for $k \sim O(1)$ and $A_{\ominus} \sim O\left(\epsilon^{1 / 4}\right)$.

Figure 6. Composite hydrodynamic stability boundary for $A_{\Theta} \sim O\left(\epsilon^{1 / 4}\right)$. wavenumber disturbances. As in the inviscid case (Section 7), the transition from the composite hydrodynamic boundary in Figure 6 to the corresponding pulsating and cellular stability boundaries that describe the temperature-insensitive limit occurs in the regime $A_{\Theta} \sim \mathrm{O}\left(\epsilon^{1 / 2}\right)$ for wavenumbers $k \sim \mathrm{O}(\epsilon)^{1 / 2}$.

\section{Summary}

The present work has continued a series of formal asymptotic treatments of hydrodynamic instability in liquid-propellant combustion based on a generalized Landau/Levich model that allows for a dynamic dependence of the burning rate on local pressure and temperature perturbations. The focus in the present study was on the effects of thermal coupling, represented by nonzero values of an appropriately defined temperature-sensitivity parameter $A_{\ominus}$, on the nature of a pulsating stability boundary that exists for negative values of a corresponding pressure-sensitivity parameter $A_{p}$. Again exploiting the smallness of the gas-to-liquid density ratio $\rho$, which provides the underlying basis for the asymptotic treatment of the stability problem, it was shown that for sufficiently large values of $A_{\Theta}$, the pulsating boundary possesses a turning point that renders steady, planar burning intrinsically unstable for sufficiently small wavenumber perturbations.

Composite Pulsating/Cellular Stability Boundary

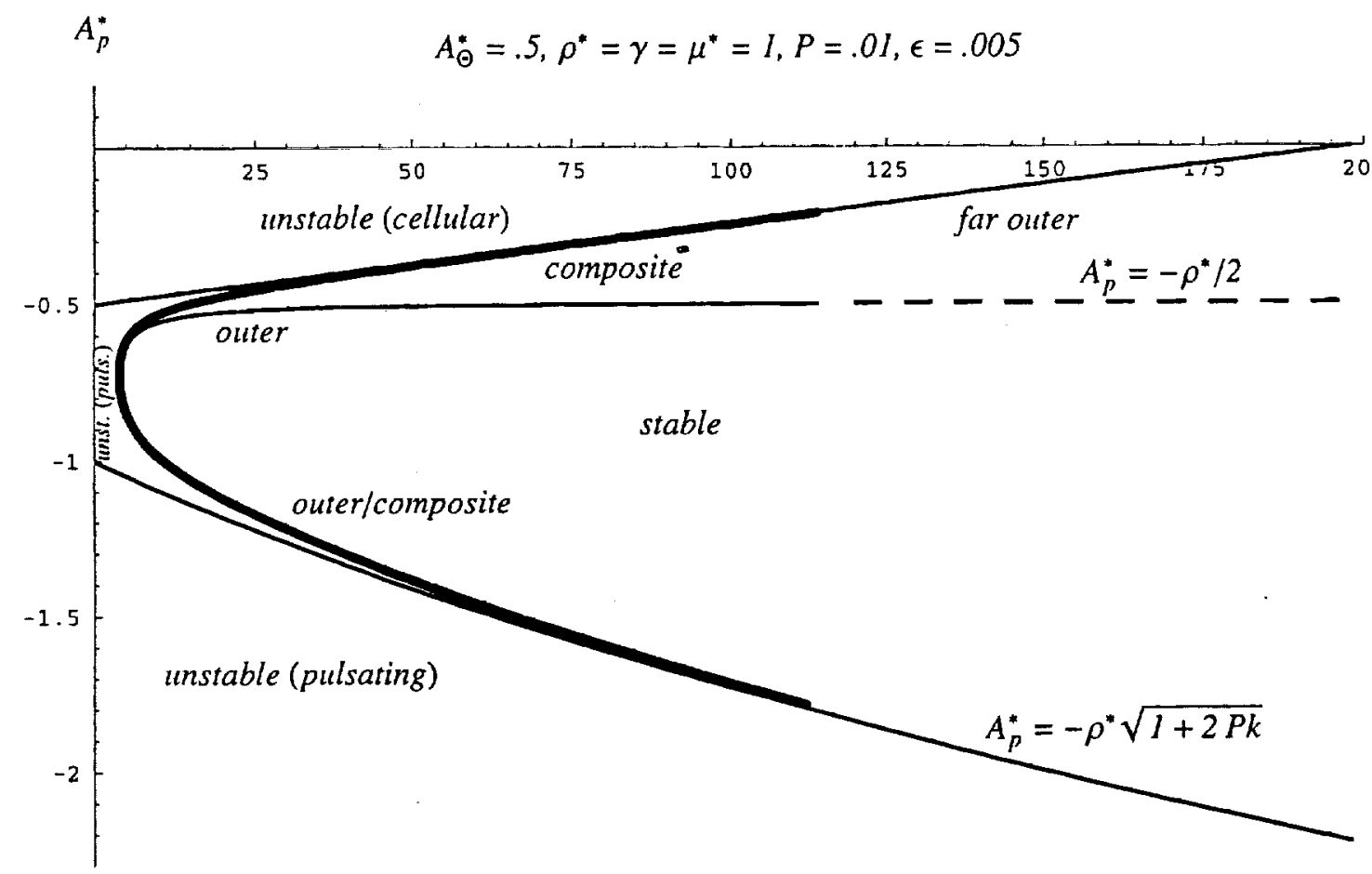


As this parameter decreases, the stable region reemerges such that in the limit that $A_{\Theta}$ vanishes, the original separated pulsating and cellular stability branches are recovered. The evolution of the pulsating boundary between these two structures was shown to occur on an intermediate wavenumber scale relative to the inner, outer and far outer wavenumber scales that emerge in the asymptotic limit of small density ratios in the absence of thermal coupling. Values of the ratio $A_{\ominus} / A_{p}$ over which this evolution occurs were shown to be roughly of the order of the overall activation energy, suggesting that a pulsating, rather than the classical Landau (cellular), form of hydrodynamic instability may be the more likely manifestation of hydrodynamic instability in at least some types of liquid propellants.

\section{Acknowledgments}

This work was supported by the United States Department of Energy under contract DE-AC04 -94 AL 85000 and by the NASA Microgravity Science Research Program under contract C-32031-E.

\section{References}

[1] Landau, L. D., On the Theory of Slow Combustion, Acta Physicochimica URSS 19:77-85 (1944), Zh. Eksp. i Teor. Fiz. 14:240 (1944).

[2] Matalon, M., and Matkowsky, B. J., Flames as Gasdynamic Discontinuities, J. Fluid Mech. 124:239-259 (1982).

[3] Pelcé, P., and Clavin, P., Influence of Hydrodynamics and Diffusion Upon the Stability Limits of Laminar Premixed Flames, J. Fluid Mech. 124:219-238 (1982).

[4] Levich, V. G., On the Stability of the Flame Front When a Liquid is Burning Slowly, Dokl. Akad. Nauk SSSR 109:975-978 (1956).

[5] Markstein, G. H., Non-Steady Flame Propagation, Pergamon Press, Oxford, 1964.

[6] Sivashinsky, G. I., Diffusional-Thermal Theory of Cellular Flames, Combust. Sci. Tech. 15: 137-146 (1977).

[7] Margolis, S. B., The Transition to Nonsteady Deflagration in Gasless Combustion, Prog. Energy Combust. Sci. 17:135-162 (1991).

[8] Margolis, S. B., and Williams, F. A., Diffusional-Thermal Coupling and Intrinsic Instability of Solid Propellant Combustion, Combust. Sci. Tech. 59:27-84 (1988).
[9] Margolis, S. B., and Williams, F. A., Diffusional/Thermal Instability of a Solid Propellant Flame, SIAM J. Appl. Math. 49:1390-1420 (1989).

[10] Bechtold, J. K., and Margolis, S. B., Hydrodynamic Stability of Solid and Liquid Propellant Combustion, Combust. Sci. Tech. 68:49-84 (1989).

[11] Armstrong, R. C., and Margolis, S. B., Hydrodynamic and Reactive/Diffusive Instabilities in a Dynamic Model of Liquid Propellant Combustion, Twenty-Second Symposium (International) on Combustion, The Combustion Institute, Pittsburgh, pp. 1807-1815 (1989).

[12] Armstrong, R. C., and Margolis, S. B., Hydrodynamic and Reactive/Diffusive Instabilities in a Dynamic Model of Liquid Propellant Combustion - II. Inviscid Fluid Motions, Combust. Flame 77:123-138, (1989).

[13] Margolis, S. B., Hydrodynamic Instability in an Extended Landau/Levich Model of LiquidPropellant Combustion at Normal and Reduced Gravity, Combust. Flame 113:406-423 (1998).

[14] Margolis, S. B., On Pulsating and Cellular Forms of Hydrodynamic Instability in LiquidPropellant Combustion, Twenty-Seventh Symposium (International) on Combustion, $\mathrm{pp}$. 2375-2386 (1998).

[15] Vosen, S. R., The Burning Rate of Hydroxylammonium Nitrate Based Liquid Propellants, Twenty-Second Symposium (International) on Combustion, The Combustion Institute, Pittsburgh, pp. 1817-1825 (1989).

[16] Bechtold, J. K., and,Margolis, S. B., Nonlinear Hydrodynamic Stability and Spinning Deflagration of Liquid Propellants, SIAM J. Appl. Math. 51:1356-1379 (1991).

[17] Timnat, Y. M., Advanced Chemical Rocket Propulsion, Academic Press, London, 1987.

[18] Margolis, S. B., Pulsating Hydrodynamic Instability and Thermal Coupling in an Extended Landau/Levich Model of Liquid-Propellant Combustion - I. Inviscid Analysis, to appear (1999).

[19] Margolis, S. B., Pulsating Hydrodynamic Instability and Thermal Coupling in an Extended Landau/Levich Model of Liquid-Propellant Combustion - II. Viscous Analysis, to appear (1999). 